\title{
Identification and characterization of a novel zebrafish (Danio rerio) pentraxin-carbonic anhydrase
}

\author{
Maarit S Patrikainen ${ }^{1}$ ， Martti E E Tolvanen ${ }^{\text {Corresp., }}{ }^{2}$, Ashok Aspatwar ${ }^{1,3}$ ， Harlan R Barker ${ }^{1}$, Csaba Ortutay ${ }^{4}$, \\ Janne Jänis ${ }^{5}$, Mikko Laitaoja ${ }^{5}$, Vesa P Hytönen ${ }^{1,3}$, Latifeh Azizi ${ }^{1}$, Prajwol Manandhar ${ }^{1,6}{ }$, Edit Jáger ${ }^{7}$, \\ Daniela Vullo $^{8}$, Sampo Kukkurainen ${ }^{1}$, Mika Hilvo ${ }^{1,9}$, Claudiu T Supuran ${ }^{8}$, Seppo Parkkila ${ }^{1,3}$ \\ 1 Faculty of Medicine and Life Sciences, University of Tampere, Tampere, Finland \\ 2 Department of Future Technologies, University of Turku, Turku, Finland \\ 3 Fimlab Ltd and Tampere University Hospital, Tampere, Finland \\ 4 HiDucator Ltd., Kangasala, Finland \\ 5 Department of Chemistry, University of Eastern Finland, Joensuu, Finland \\ 6 Center for Molecular Dynamics Nepal, Kathmandu, Nepal \\ 7 Faculty of Health Sciences, Department of Epidemiology, Semmelweis University, Budapest, Hungary \\ 8 Dipartimento Neurofarba, Sezione di Scienze Farmaceutiche e Nutraceutiche, Università degli Studi di Firenze, Florence, Italy \\ 9 Zora Biosciences Ltd, Espoo, Finland \\ Corresponding Author: Martti E E Tolvanen \\ Email address: martti.tolvanen@utu.fi
}

Background. Carbonic anhydrases (CA) are ubiquitous, essential enzymes which catalyze the conversion of carbon dioxide and water to bicarbonate and $\mathrm{H}^{+}$ions. Vertebrate genomes generally contain gene loci for 15 to 21 different CA isoforms, three of which are enzymatically inactive. CA VI is the only secretory protein of the enzymatically active isoforms. We discovered that non-mammalian carbonic anhydrase VI contains a C-terminal pentraxin domain, a novel combination for both CAs and pentraxins.

Methods. We isolated and sequenced zebrafish (Danio rerio) CA VI cDNA, complete with the sequence coding for the pentraxin (PTX) domain, and produced the recombinant CA VI-PTX protein. Enzymatic activity and kinetic parameters were measured with a stopped-flow instrument. Mass spectrometry, analytical gel filtration and dynamic light scattering were used for biophysical characterization. Sequence analyses and Bayesian phylogenetics were used in generating hypotheses of protein structure and CA VI gene evolution. A CA VI-PTX antiserum was produced, and the expression of CA VI protein was studied by immunohistochemistry. A knock-down zebrafish model was constructed, and larvae were observed up to 5 days post-fertilization. The expression of ca6 mRNA was quantitated by qRT-PCR in different developmental times in morphant and wild-type larvae and in different adult fish tissues. Finally, the swimming behavior of the morphant fish was compared to that of wild-type fish.

Results. The recombinant enzyme has a very high carbonate dehydratase activity. Sequencing confirms a 530-residue protein identical to one of the predicted proteins in the Ensembl database (ensembl.org). The protein is pentameric in solution, as studied by gel filtration and light scattering, presumably joined by the pentraxin domains. Mass spectrometry confirms the predicted signal peptide cleavage and disulfides, and $\mathrm{N}$-glycosylation in two of the four observed glycosylation motifs. Molecular modelling of the pentamer is consistent with the modifications observed in mass spectrometry. Phylogenetics and sequence analyses provide a consistent hypothesis of the evolutionary history of domains associated with CA VI in mammals and non-mammals. Briefly, the evidence suggests that ancestral CA VI was a transmembrane protein, the exon coding for the cytoplasmic domain was replaced by one coding for PTX 
domain, and finally, in the therian lineage, the PTX-coding exon was lost. We knocked down CA VI expression in zebrafish embryos with antisense morpholino oligonucleotides, resulting in phenotype features of decreased buoyancy and swim bladder deflation in $4 \mathrm{dpf}$ larvae.

Discussion. These findings provide novel insights into the evolution, structure, and function of this unique $\mathrm{CA}$ form. 


\section{Identification and characterization of a novel \\ 2

4

5 


\section{ABSTRACT}

Background. Carbonic anhydrases (CA) are ubiquitous, essential enzymes which catalyze the conversion of carbon dioxide and water to bicarbonate and $\mathrm{H}^{+}$ions. Vertebrate genomes generally contain gene loci for 15 to 21 different $\mathrm{CA}$ isoforms, three of which are enzymatically inactive. CA VI is the only secretory protein of the enzymatically active isoforms. We discovered that non-mammalian carbonic anhydrase VI contains a C-terminal pentraxin domain, a novel combination for both CAs and pentraxins.

Methods. We isolated and sequenced zebrafish (Danio rerio) CA VI cDNA, complete with the sequence coding for the pentraxin (PTX) domain, and produced the recombinant CA VI-PTX protein. Enzymatic activity and kinetic parameters were measured with a stopped-flow instrument. Mass spectrometry, analytical gel filtration and dynamic light scattering were used for biophysical characterization. Sequence analyses and Bayesian phylogenetics were used in generating hypotheses of protein structure and CA VI gene evolution. A CA VI-PTX antiserum was produced, and the expression of CA VI protein was studied by immunohistochemistry. A knock-down zebrafish model was constructed, and larvae were observed up to 5 days postfertilization. The expression of ca6 mRNA was quantitated by qRT-PCR in different developmental times in morphant and wild-type larvae and in different adult fish tissues. Finally, the swimming behavior of the morphant fish was compared to that of wild-type fish.

Results. The recombinant enzyme has a very high carbonate dehydratase activity. Sequencing confirms a 530-residue protein identical to one of the predicted proteins in the Ensembl database (ensembl.org). The protein is pentameric in solution, as studied by gel filtration and light scattering, presumably joined by the pentraxin domains. Mass spectrometry confirms the predicted signal peptide cleavage and disulfides, and N-glycosylation in two of the four observed glycosylation motifs. Molecular modelling of the pentamer is consistent with the modifications observed in mass spectrometry. Phylogenetics and sequence analyses provide a consistent hypothesis of the evolutionary history of domains associated with CA VI in mammals and nonmammals. Briefly, the evidence suggests that ancestral CA VI was a transmembrane protein, the exon coding for the cytoplasmic domain was replaced by one coding for PTX domain, and finally, in the therian lineage, the PTX-coding exon was lost. We knocked down CA VI expression in zebrafish embryos with antisense morpholino oligonucleotides, resulting in phenotype features of decreased buoyancy and swim bladder deflation in 4 dpf larvae.

Discussion. These findings provide novel insights into the evolution, structure, and function of this unique $\mathrm{CA}$ form.

\section{INTRODUCTION}

Carbonic anhydrase VI (CA VI) is the only secretory CA enzyme in mammals. In its very first reporting, Henkin et al. described a novel protein, gustin, from human saliva (Henkin et al., 1975), which was later shown to be CA VI (Thatcher et al., 1998). This protein was first described as a CA enzyme by Fernley et al. (Fernley, Wright \& Coghlan, 1979), who identified a novel high molecular weight form of CA in the sheep parotid gland and saliva. The first immunohistochemical studies on human CA VI indicated that it is highly expressed in the serous acinar cells of the parotid and submandibular glands (Parkkila et al., 1990). It is one of the major protein constituents of human saliva (Parkkila et al., 1993), and also found in human and rat milk (Karhumaa et al., 2001). 
The physiological role of CA VI has remained unclear, even though it was discovered three decades ago. Henkin's group linked gustin (CA VI) to the regulation of taste function (Shatzman, Henkin, 1981). Expression of CA VI in the von Ebner's glands implicate CA VI in the paracrine modulation of taste function and TRC apoptosis (Leinonen et al., 2001). Various studies have later shown a link between bitter taste perception and CA VI, e.g. (Melis et al., 2013; Patrikainen et al., 2014). Two studies have shown a link between CA VI and immunological function in mouse and human. First, Car $^{-/-}$mice have a greater number of lymphoid follicles in the small intestinal Peyer's patches, suggesting an immunological phenotype (Pan et al., 2011). Second, the analysis of gene expression in the trachea and lung of $\mathrm{Car}^{-/-}$mice showed alterations in biological processes such as antigen transfer to mucosal-associated lymphoid tissue (Patrikainen et al., 2016).

Innate immune systems, based on pattern recognition, exist in some form in all metazoan organisms (Medzhitov, 2007). The pattern-recognition molecules (PRMs) recognize conserved structures on the surface of pathogens and activate the innate immune response. Pentraxins are a superfamily of fluid phase pattern recognition molecules conserved in evolution and characterized by a cyclic multimeric structure with a regulatory role in inflammation (Bottazzi et al., 2016). They contain a characteristic 200-residue-long domain at their C-terminus. Based on their primary subunit structures pentraxins are divided into short pentraxins and long pentraxins. Short pentraxins are classically represented by C-reactive protein (a.k.a. CRP, pentraxin-1, PTX1) and serum amyloid P (a.k.a. APCS, SAP, pentraxin-2, PTX-2), whereas long pentraxins comprise pentraxin-3 (PTX3), neuronal pentraxins, and others (Garlanda et al., 2005).

We noted the presence of an additional PTX domain in some non-mammalian CA6 gene predictions in 2007, but did not follow up this observation at that time. More recently, with more non-mammalian genomes available, we realized that the PTX domain is present in nonmammalian CA VI too consistently to be an annotation artifact, which inspired this study. We used zebrafish (Danio rerio) as a vertebrate model organism for functional and structural characterization of the PTX-associated CA VI.

\section{MATERIALS AND METHODS}

\section{Sequence conservation}

In order to compare conservation in the CA and PTX domains of CA VI-PTX proteins, nonmammalian CA VI sequences were retrieved from NCBI (NCBI Resource Coordinators, 2016) nr protein database as of Dec $5^{\text {th }}, 2015$, using blastp (https://blast.ncbi.nlm.nih.gov/Blast.cgi?PAGE=Proteins) (Altschul et al., 1990), with human CA VI (ENSP00000366662 from Ensembl, (Flicek et al., 2012) as query sequence, taxonomically filtered for non-mammalian vertebrates. Full-length or nearly full-length CA VI-PTX sequences were seen at extremely low e values, not higher than $2 \times 10^{-80}$, indicating very high similarity; CA VI sequence fragments were seen at e values from $10^{-79}$ to $10^{-71}$; and the remaining matches, at $\mathrm{e}$ values of $10^{-68}$ and higher, were annotated as other CA isoforms and did not contain a pentraxin domain. Sequences with an e value of $2 \times 10^{-80}$ or lower were taken for further quality control. We discarded sequences shorter than 485 residues and any with non-specific " $\mathrm{X}$ " characters. 
121 Furthermore, we rejected sequences with unaligned, unique insertions of at least 20 residues at exon boundaries, which we assume to be introns mispredicted as coding sequence. Likewise, sequences containing gaps in the alignment between exon boundaries were interpreted to miss data for internal exons and were discarded. Thus, all sequences which were incomplete in the CA domain were discarded, but sequences devoid of the signal peptide region were still kept. The final sequence set contained 78 sequences from 75 species, (sequence accession numbers shown in Fig. S1 and in Data S1). After inspection of the multiple sequence alignment, four sequences were edited for a more plausible initiation site (Table 1) deemed to be at the conserved $\mathrm{M}$ at the start of the signal peptide region. All sequences had complete PTX domains. Sequences were aligned with Clustal Omega (Sievers et al., 2011). In order to calculate conserved positions in each domain, the CA domain was defined to correspond to residues 24 to 280 in zebrafish CA VI (UniProt annotation in E9QB97_DANRE), and the PTX domain was defined as residues 317 to 518 (InterProScan at http://www.ebi.ac.uk/interpro/sequence-search (Jones et al., 2014), match to profile SM00159, PTX).

\section{Phylogenetic analyses}

For the tree in Fig. 1, cDNA sequences and their protein translations were collected from the Ensembl database (release 67) for carbonic anhydrases 6, 9, 12, and 14, from selected species. Protein sequences were aligned with Clustal Omega. Codon aligned cDNA sequences were produced in the PAL2NAL web server v. 14 (http://www.bork.embl.de/pal2nal/) (Suyama, Torrents \& Bork, 2006) using the protein alignment as a guide (protein alignment: Data S2; final codon alignment: Data S3). For the tree in Fig. S2, a second alignment was similarly made using catalytic domains of CA VI sequences only (protein alignment: Data S4; final codon alignment: Data S5). For the tree in Fig. 2, we made a third alignment of CA VI-associated pentraxin domains from selected species and human pentraxins (codon aligned sequences: Data S6). The resulting codon (DNA) alignments and the program MrBayes v 3.2 (Ronquist et al., 2012) were used to estimate the phylogeny of the sequences by Bayesian inference. Bayesian estimation was run for at least 10,000 generations, with flat a priori distribution of base frequencies, substitution rates, proportion of invariable sites, and gamma shape parameter. The $50 \%$ majority rule consensus trees were saved and visualized using the APE R package (Paradis, Claude \& Strimmer, 2004).

Run lengths, relevant parameters at the end of run, and rooting of the trees were as follows. For the first tree, the average standard deviation of split frequencies after 10,000 generations was 5.2 $\mathrm{x} 10^{-2}$ when the analysis was stopped. The arithmetic mean of the estimated marginal likelihoods for runs sampled was -17175.07. Drosophila melanogaster CAH1 sequence was used as an outgroup to root the tree. For the second tree, the average standard deviation of split frequencies after 20,000 generations was $1.2 \times 10^{-1}$ when the analysis was stopped. The arithmetic mean of the estimated marginal likelihoods for runs sampled was -10596.5. Fish sequences were used as an outgroup to root the consensus tree. Branching points with lower than $50 \%$ consensus in the mammal branch are collapsed. Finally, for the third tree, the average standard deviation of split frequencies after 10,000 generations was $8.1 \times 10^{-2}$ when the analysis was stopped. The arithmetic mean of the estimated marginal likelihoods for runs sampled was -10319.1. 


\section{BlastN search in platypus genome}

In order to see if the orphan fragment Contig22468 of platypus genome (which contains the exon coding for a "CA VI-type" PTX domain) would have been somehow missed in the genome assembly, we performed a BlastN search in Ensembl (http://www.ensembl.org/Homo_sapiens/Tools/Blast?db=core). BlastN was run against the platypus genome with the full $11,311 \mathrm{nt}$ sequence of supercontig:OANA5:Contig22468 as query sequence.

\section{Exon length comparisons}

Exon data was retrieved from Ensembl. Lengths of the exons that follow those coding for the CA domain were noted for Ensembl transcripts for human CA6 (ENST00000377443), CA9 (ENST00000378357), CA12 (ENST00000178638 and ENST00000344366), and CA14 (ENST00000369111), and zebrafish ca6 (ENSDART00000132733). Similarly, lengths of the exons preceding the PTX domain exon were noted for Ensembl transcripts of human CRP (ENST00000255030), APCS (SAP, ENST00000255040).

\section{Amphipathic helix prediction}

A study of the region between the CA and PTX domains in the alignment of CA VI protein sequences showed little conservation except for five sites with hydrophobic residues spaced 3 or 4 residues apart, with mostly polar residues between them, suggestive of an amphipathic alpha helix. The subsequences from 287 to 303 and from 293 to 309 for human and zebrafish CA VI, respectively, were visualized as helical wheel diagrams, or end projections of a hypothetical alpha helix of 17 residues, using the PepWheel program of the EMBOSS suite (http://www.bioinformatics.nl/cgi-bin/emboss/help/pepwheel) (Rice, Longden \& Bleasby, 2000).

\section{Construction of recombinant baculoviruses}

The 1593-bp zebrafish ca6 sequence encoding the full-length, pentraxin-containing CA VI polypeptide (CA VI-PTX) was amplified by PCR using the forward primer 5'ATGGAGCAGCTGACTCTAGTC-3' and reverse primer 5'TTTCTCTGTTTCTCTATTATTATTAT-3'. PCR conditions consisted of an initial denaturation step at $98{ }^{\circ} \mathrm{C}$ for $30 \mathrm{sec}$ followed by 35 cycles at $98{ }^{\circ} \mathrm{C}$ for $10 \mathrm{sec}$ (denaturation), $55^{\circ} \mathrm{C}$ for 30 sec (annealing), and $72{ }^{\circ} \mathrm{C}$ for $25 \mathrm{sec}$ (elongation). The final extension step was carried out at 72 ${ }^{\circ} \mathrm{C}$ for $5 \mathrm{~min}$. The expression construct was optimized for protein production in Spodoptera frugiperda insect cells $(\mathrm{Sf} 9)$ by inserting into second round $\mathrm{PCR}$ primers restriction sites for BamHI and SalI plus sequences coding for C-terminal histidine tag for protein purification and a thrombin cleavage site for tag removal. The second round of PCR was carried out using the protocol described above, except that the temperature for annealing was $62{ }^{\circ} \mathrm{C}$, and final extension step was carried out at $74{ }^{\circ} \mathrm{C}$ for 7 minutes. The baculoviral genomes encoding CA VI recombinant proteins were generated according to the Bac-To-Bac Baculovirus Expression System instructions (Invitrogen). The recombinant protein insert was sequenced using ABI PRISM BigDye ${ }^{\circledR}$ Terminator v3.1 Cycle Sequencing kit (Applied Biosystems Inc.) and pFASTBac primers (forward: 5'-AATGATAACCATCTGGCA-3' and reverse: 5'- 
212 GGTATGGCTGATTATGAT-3') in order to obtain the full-length insert sequence. The PCR

213 conditions consisted of 35 cycles at $96{ }^{\circ} \mathrm{C}$ for $10 \mathrm{sec}$ (denaturation), $50{ }^{\circ} \mathrm{C}$ for $5 \mathrm{sec}$ (annealing),

214 and $55^{\circ} \mathrm{C}$ for $4 \mathrm{~min}$ (elongation) with final extension at $37{ }^{\circ} \mathrm{C}$ for $5 \mathrm{~min}$.

215

216

217

218

219

220

221

222

223

224

225

226

227

228

229

230

\section{Production and purification of recombinant CA VI-PTX}

The Sf9 insect cells (Invitrogen) were maintained in HyQ SFX-Insect serum-free cell culture medium (HyClone, Logan, UT). The cells were centrifuged $\left(2000 \mathrm{X} \mathrm{g}\right.$, at $20{ }^{\circ} \mathrm{C}$, for $\left.5 \mathrm{~min}\right) 72 \mathrm{~h}$ after infection, and the medium was collected. Purification was performed with the Probond ${ }^{\circledR}$ Purification System (Invitrogen) under native binding conditions with wash and elution buffers made according to the manufacturer's instructions. Purity of the protein was checked and the molecular weight (MW) of the recombinant protein was determined by running a $10 \%$ SDSPAGE (sodium dodecyl sulphate polyacryl amide gel electrophoresis) under reducing conditions. The size of the protein was determined using Precision Plus Protein ${ }^{\mathrm{TM}}$ Standards Dual Color (Bio-Rad Laboratories, Inc., CA, USA.) and molecular weight marker and bands were visualized using the Colloidal Blue Staining Kit ${ }^{\mathrm{TM}}$ (Invitrogen).

\section{Carbonic anhydrase activity/inhibition assay}

An Applied Photophysics stopped-flow instrument was used for assaying the CA catalyzed $\mathrm{CO}_{2}$ hydration activity (Khalifah, 1971). The method was exactly as described previously (Berrino et al., 2017) except that the inhibitor dilutions were done up to $0.5 \mathrm{nM}$.

\section{Light scattering experiments}

Molecular weight determination of zebrafish CA VI-PTX was performed using a Malvern Zetasizer $\mu \mathrm{V}$ instrument (Malvern Instruments Ltd, Worcestershire, UK) running Static Light Scattering (SLS) and Dynamic Light Scattering (DLS) methods. Analysis was performed using a liquid chromatography instrument (CBM-20A, Shimadzu Corporation, Kyoto, Japan) equipped with autosampler (SIL-20A), UV-VIS (SPD-20A) and fluorescence detector (RF-20Axs). Data were processed using Lab Solution Version 5.51 (Shimadzu Corporation) and OmniSec 4.7 (Malvern Instruments) softwares. A sample of the protein $(50 \mu \mathrm{g})$ was injected on a Superdex $2005 / 150$ column (GE Healthcare, Uppsala, Sweden) equilibrated with $50 \mathrm{mM} \mathrm{NaH}_{3} \mathrm{PO}_{4}, 500$ $\mathrm{mM} \mathrm{NaCl} \mathrm{pH} 8$ buffer. Runs were performed with flow rate of $0.1 \mathrm{ml} / \mathrm{min}$ at $20^{\circ} \mathrm{C}$ using a thermostated cabin. The molecular weight of the zebrafish CA VI-PTX was determined either by using a standard curve based on molecular weight standard proteins (SEC analysis; carbonic anhydrase $29 \mathrm{kDa}$, alcohol dehydrogenase $150 \mathrm{kDa}, \beta$-amylase $200 \mathrm{kDa}$, BSA $66 \mathrm{kDa}$, SigmaAldrich) or by calibrating the light scattering detector using the monomeric peak of BSA and light-scattering intensity (SLS).

\section{Sample preparation for mass spectrometry}

Prior to the mass spectrometric measurements, the CA VI-PTX sample was buffer-exchanged to $10 \mathrm{mM}$ ammonium acetate ( $\mathrm{pH}$ 7.5) buffer using Sephadex G-25 M (PD-10) desalting columns (GE Healthcare, Gillingham, UK). Ten 1-mL fractions were collected, and the fractions containing protein were concentrated using Amicon Ultra (5-kDa cut-off) centrifugal filter 
258

259

260

261

262

263

264

265

266

267

268

269

270

271

272

273

274

275

276

277

278

279

280

281

282

283

284

285

286

287

288

289

290

291

292

293

294

295

296

297

298

299

300

301

302

303

devices (Merck Millipore, Darmstadt, Germany). Finally, protein concentrations were determined by UV-absorbance at $280 \mathrm{~nm}$, using a sequence-derived extinction coefficient 99155 $\mathrm{M}^{-1} \mathrm{~cm}^{-1}$, calculated by ProtParam at http://web.expasy.org/protparam/ (Gasteiger et al., 2003). Intact protein mass analysis was performed by diluting the sample to the desired protein concentration with acetonitrile $(\mathrm{MeCN})$, containing $1 \%$ of acetic acid (HOAc). Alternatively, CA VI-PTX was digested with trypsin. Briefly, an aliquot of the CA VI-PTX sample was mixed with a sequencing grade modified trypsin (Promega, Madison, WI, USA) ( $3 \mathrm{mg} / \mathrm{mL}$ in water) to obtain a 1:20 (w/w) protease-to-protein ratio. The digest sample was incubated at $37^{\circ} \mathrm{C}$ for $1 \mathrm{~h}$, and subsequently diluted to approximately $10 \mu \mathrm{M}$ with $\mathrm{MeCN}$ containing $1 \% \mathrm{HOAc}$.

\section{Mass measurements and data analysis}

All experiments were performed on a 12-T Bruker Solarix-XR FT-ICR mass spectrometer (Bruker Daltonik GmbH, Bremen, Germany), equipped with an Apollo-II electrospray ionization (ESI) source and a dynamically harmonized ParaCell ICR-cell. All protein/peptide samples were directly infused into the ESI source at a flow rate of $1.5 \mu \mathrm{L} / \mathrm{min}$. The ESI-generated ions were externally accumulated in the hexapole collision cell for $1 \mathrm{~s}$, and transferred to the ICR cell for trapping, excitation and detection. For each mass spectrum, a total of 300 time-domain transients (1 MWord each) were co-added, and zero-filled once to obtain final 2 MWord broadband data. For collision-induced dissociation tandem mass spectrometry (CID-MS/MS) experiments, the precursor ions of interest were mass-selected in a quadrupole and fragmented in the collision cell by increasing the collision voltage to the appropriate value. The mass spectra were externally calibrated with ESI-L Low concentration tuning mix (Part n:o G1969-85000; Agilent Technologies, Santa Clara, CA, USA). The instrument was operated and the data were acquired by using Bruker ftmsControl 2.0 software. The mass spectra were subsequently transferred to Bruker DataAnalysis 4.4 software for further processing. Spectral de-isotoping and charge-state deconvolution (to obtain monoisotopic peptide masses) was accomplished with a Bruker SNAP2 peak-picking module. The obtained mass lists were then uploaded to GPMAW 10.0 software (Lighthouse Data, Odense, Denmark) for tryptic peptide identification. Only specific tryptic peptides were searched with a maximum mass error of $5 \mathrm{ppm}$. The glycosylation sites in CA VIPTX were identified by incorporating typical high-mannose/complex glycans with a various number of residues into the four putative $\mathrm{N}$-glycosylation sites and searched against the obtained mass lists.

\section{Homology modeling of zebrafish CA VI}

We built a 3D model of zebrafish CA VI starting from PDB 3FE4, human CA VI (Pilka et al., 2012); and 4AVS, human Serum Amyloid P Component, SAP (Kolstoe et al., 2014), as templates for the CA domain and PTX domain, respectively. The CA template includes residues 32 to 280 of human CA VI, missing 14 residues in the N-terminus of the mature protein, and 28 residues in the C-terminus. Briefly, the predicted amphipathic helix (APH) region of human CA VI (287 to 303) and four additional residues (283 to 286) were modelled as an alpha helix, and the helix was subsequently docked to the C-terminal face of 3FE4. This extended model of human CA VI was used as a template in homology modeling the CA domain plus APH of zebrafish CA VI. The model of the PTX domain of zebrafish CA VI was docked to the model of $\mathrm{CA}+\mathrm{APH}$ domains. Finally, a pentameric model of CA VI-PTX was created by superimposing 
304 five copies of the monomer model over each monomer in the pentameric SAP structure (PDB 305 4AVS).

306

307

The C-terminal alpha helix was generated automatically ab initio for the region predicted to form an APH when the full sequence of human CA VI was given as a modelling target to I-TASSER 4.0 (Roy, Kucukural \& Zhang, 2010). The helix was separated from the model and subsequently docked to 3FE4, using the HADDOCK 2.1 server (http://haddock.science.uu.nl/) (de Vries et al., 2007). A list of potential interface residues in 3FE4 were predicted through CPORT (http://milou.science.uu.nl/services/CPORT/) (de Vries, Bonvin, 2011), and only those lying on the C-terminal face of 3FE4 were set as interacting residues in docking. For the APH, the residues located on the hydrophobic side were chosen as interacting residues. The resulting model from this docking step was used as a template to model the full CA domain of zebrafish CA VI at the MODELLER server (Webb, Sali, 2016) using UCSF Chimera v. 1.10 (Pettersen et al., 2004) as the interface program. The PTX domain of zebrafish CA VI (residues 317-530) was also modelled by MODELLER, with 4AVS as template. The two partial models of zebrafish CA VI were joined by docking with HADDOCK, again predicting interacting residues with CPORT. Most of the predicted interacting residues were located near the C-terminus of the CA domain and near the N-terminus of the PTX domain, and the residues in these regions were chosen as active residues in docking. The structural superimpositions of the PTX domains of the CA VIPTX monomer on the SAP pentamer (4AVS) were carried out with the MatchMaker tool in UCSF Chimera to generate the final pentamer model.

\section{Zebrafish maintenance and ethical permissions}

Wild type zebrafish of the $\mathrm{AB}$ strain were maintained at $28.5^{\circ} \mathrm{C}$ under standard conditions (Westerfield, 2007). We express the embryonic ages in hours post-fertilization (hpf) and days post-fertilization (dpf). Embryos/larvae were collected from the breeder tanks with a sieve and rinsed with embryonic medium (Sarsted, Nümbrecht, Germany) into Petri dishes.

Embryos/larvae were kept in Petri dishes in embryonic medium supplemented with 1-phenyl-2thiourea (Sigma-Aldrich) at $28.5^{\circ} \mathrm{C}$ until they were used in experiments. The maximum number of larvae on a $9 \mathrm{~cm}$ diameter Petri dish was 50 . Embryonic medium contained $5 \mathrm{mM} \mathrm{NaCl}, 0.17$ $\mathrm{mM} \mathrm{KCl}, 0.33 \mathrm{mM} \mathrm{CaCl}_{2}, 0.33 \mathrm{mM} \mathrm{MgSO}_{4}$ and 10-5 \% Methylene Blue (Sigma-Aldrich). Zebrafish housing and care in the Zebrafish facility of the University of Tampere have been approved by the National Animal Experiment Board of Finland, administered through the Provincial Government of Western Finland, Province Social and Health Department Tampere Regional Service Unit (permit \# LSLH-2007-7254/Ym-23). Using 5-day old zebrafish as a model organism requires no specific ethical permission, neither does studying tissues collected from euthanized adult fish.

\section{Morpholino injections of zebrafish embryos}

Knockdown of $c a 6$ was carried out using two different antisense morpholino oligonucleotides (MOs) (GeneTools LLC, Philomath, OR, USA): one translation-blocking (MO1 5'CTGCCTGTGCTCTGAACTGTTTCTC-3') and the other splicing-blocking, to target intronexon boundary before exon 9 (MO2 5'- GCTTGCCTTGAGAAGGAAAGATCAT). The random 
control (RC) MOs (5'-CCTCTTACCTCAGTTACAATTTATA-3') were used as control MOs. The supplied MOs were re-suspended in sterile water at $1 \mathrm{mM}$ stock concentration. Immediately prior to injection, ca6-MOs were diluted to the intended concentration of $125 \mu \mathrm{M}$. In order to monitor injection efficiency, 0.2\% Dextran Rhodamine B and $0.1 \%$ Phenol Red (final concentrations; Sigma, Poole, UK) were included in the solution, and the final $\mathrm{KCl}$ concentration was adjusted to $1 \mathrm{M}$. One $\mathrm{nl}$ of antisense $\mathrm{MO}$ solution was injected into the yolk of approximately 500 one- to two-cell stage embryos, without randomization. The MO-injected embryos were screened for the presence of fluorescence after $24 \mathrm{~h}$ to select the true ca6 morphants using Lumar V1.1 fluorescence stereomicroscope (Carl Zeiss MicroImaging GmbH) and AxioVision software version 4.9. The non-fluorescent embryos were eliminated.

\section{Microscopy and live image analysis of zebrafish phenotypes}

Gross phenotypic appearance was analyzed by light-field microscopy. For each experiment, typically 10 to 20 ca6-MO-injected larvae were screened with a similar number of matched controls. Larvae were first euthanized using 0.05\% tricaine (Sigma-Aldrich) in embryo medium and embedded in $17 \%$ high molecular weight methyl cellulose in $15 \times 30 \mathrm{~mm}$ transparent polypropylene Petri dish for taking images of the developing embryos/larvae from $1 \mathrm{dpf}$ to $5 \mathrm{dpf}$ using Zeiss Stereo Microscope (Carl Zeiss MicroImaging GmbH; Göttingen, Germany) with NeoLumar S 1.5x Objective (Carl Zeiss MicroImaging GmbH). The images were analyzed with AxioVision software version 4.9. and scale bars were inserted. Images were cropped and assembled into composite images.

\section{Isolation of total RNA and synthesis of cDNA}

Total RNA was isolated at different stages of development, from 0-168 hpf whole embryos/larvae, and from different organs of the adult zebrafish. Total RNA was isolated from $30-\mu \mathrm{g}$ samples using the RNeasy ${ }^{\circledR}$ Mini kit (Qiagen, Hilden, Germany) by following the manufacturer's instructions. The concentration and purity of total RNA were determined using a Nanodrop UV/VIS Spectrophotometer at 260 and $280 \mathrm{~nm}$. Reverse transcriptase PCR was performed using 0.1-5 $\mu \mathrm{g}$ of total RNA to synthesize the first strand cDNA using First Strand cDNA Synthesis kit (High-Capacity cDNA Reverse Transcription Kits, Applied Biosystems, Foster City, CA) with random primers and M-MuLV reverse transcriptase according to the protocol recommended by the manufacturer.

\section{Quantitative Real-Time PCR}

Quantitative Real-Time PCR (qRT-PCR) primers were designed based on the complete cDNA sequence taken from Ensembl (ENSDART00000057097), using the program Primer Express ${ }^{\circledR}$ Software v2.0 (Applied Biosystems) (forward primer 5'-

CAAACATTTATTTGCCAGCACTCC-3' and reverse primer 5'TATGTCCAATAATCTCCATCTACTCC-3'). qRT-PCR was performed using the SYBR Green PCR Master Mix Kit in an ABI PRISM 7000 Detection System ${ }^{\mathrm{TM}}$ according to the manufacturer's instructions (Applied Biosystems). The PCR conditions consisted of an initial denaturation step at $95^{\circ} \mathrm{C}$ for $10 \mathrm{~min}$ followed by 40 cycles at $95^{\circ} \mathrm{C}$ for $15 \mathrm{sec}$ (denaturation) and $60^{\circ} \mathrm{C}$ for $1 \mathrm{~min}$ (elongation). The data were analyzed using the ABI PRISM 7000 SDS $^{\mathrm{TM}}$ 
396

397

398

399

400

401

402

403

404

405

406

407

408

409

410

411

412

413

414

415

416

417

418

419

420

421

422

423

424

425

426

427

428

429

430

431

432

433

434

435

436

437

438

439

440

441

software (Applied Biosystems). Every PCR was performed in a total reaction volume of $15 \mu \mathrm{l}$ containing $2 \mu \mathrm{l}$ of first strand cDNA (20 ng cDNA), $1 \times$ Power SYBR green PCR Master Mix ${ }^{\mathrm{TM}}$ (Applied Biosystems, Foster City, CA, USA), and $0.5 \mu \mathrm{M}$ of each primer. We performed these experiments in duplicate and with sample duplicates. The results of $c a 6$ gene expression were normalized using zebrafish housekeeping gene gapdh as internal control. The final results are given as relative expression values, calculated according to the Pfaffl's equation (Pfaffl, 2001).

\section{Preparation of zebrafish tissues}

The adult zebrafish were euthanized by keeping them in $1 \%$ tricaine on ice for more than 10 min followed by decapitation. Different organs were harvested under the microscope and immediately transferred them to $1.5 \mathrm{ml}$ microcentrifuge tube containing RNAlater ${ }^{\circledR}$ (Ambion, Austin, TX, USA) and were stored at $-20^{\circ} \mathrm{C}$ until further analysis. Simultaneously tissues for immunohistochemical analysis were harvested and immediately fixed with 4\% PFA for $24 \mathrm{~h}$ at 4 ${ }^{\circ} \mathrm{C}$. Tissues were transferred to $20 \%$ sucrose in PBS and stored at $4^{\circ} \mathrm{C}$ until embedding them in Tissue-Tek ${ }^{\circledR}$ O.C.T. ${ }^{\text {TM }}$ Compound (Sakura Finetek Europe B.V., Alphen aan den Rijn, The Netherlands). Embedded tissue samples were stored at $-20^{\circ} \mathrm{C}$ until further analysis.

\section{Antibody testing}

Antibody against zebrafish CA VI-PTX was manufactured by Innovagen AB (Innovagen AB, Lund, Sweden) according to their standard immunization schedule, with boosters at 14, 28, 49, and 70 days. Pre-immune serum and three samples of polyclonal antiserum were tested using dot blotting. Bio-Dot ${ }^{\circledR}$ Microfiltration Apparatus (BioRad) was used to attach $500 \mathrm{ng}$ of produced and purified native zebrafish CA VI-PTX protein to PROTRAN® nitrocellulose (NC) transfer membrane (Schleicher \& Schuell GmbH, Dassel, Germany) according to manufacturer's instructions. Prior to staining, nonspecific binding of the primary antibody was prevented using diluted colostrum (1:10 in Tris-Buffered Saline with Tween 20 [TBST]) as a blocking agent for 30 minutes. Pre-immune serum, bleed 1 (day 41), bleed 2 (day 62), and bleed 3 (day 83) of polyclonal rabbit anti-zebrafish CAVI-PTX (Innovagen AB), diluted 1:100 in TBST, were added to $\mathrm{NC}$ strips which were incubated at room temperature for $1 \mathrm{~h}$. Donkey anti-rabbit $\mathrm{IgG}$, horseradish peroxidase linked whole antibody (Amersham Biosciences, GE Healthcare Life Sciences, Little Chalfont, UK) diluted 1:25,000 in TBST was used as secondary antibody. Washing steps were carried out using TBST. Staining was carried out using ImmPACTTM DAB Peroxidase Substrate Kit (Vector Laboratories, Inc., Burlingame, CA, USA). The testing showed that bleed 1 and bleed 2 have a strong reactivity against zebrafish CA VI-PTX (Fig. S3 B and C). Antiserum of bleed 2 was used in further experiments.

\section{Immunohistochemistry of zebrafish tissues}

The Tissue-Tek ${ }^{\circledR}$ O.C.T. ${ }^{\text {TM }}$ Compound-embedded samples were cut into $10 \mu \mathrm{m}$ sections using cryotome and prior to staining, the sections were attached to the glass slide by incubating at 37 ${ }^{\circ} \mathrm{C}$ overnight. Staining procedure of tissue samples was carried out as described above. Alexa Fluor ${ }^{\circledR}$ goat anti-rabbit IgG 1:1000 (Life Technologies, Carlsbad, USA) was used as a secondary antibody, and sections were mounted with Vectashield Hard Set Mounting Medium with nuclear dye DAPI (4',6-diamidino-2-phenylindole, Vector Laboratories Inc., Burlingame, CA, USA). 
442 The sections were photographed using Zeiss LSM780 Laser Scanning Confocal Microscope with

443 Zeiss Cell Observer.Z1 microscope, Plan-Apochromat 40x/1.4 (oil) objective, with pulsed diode

444 laser $405 \mathrm{~nm}$ and multiline Argon laser: $488 \mathrm{~nm}$, and Quasar spectral GaAsP PMT array detector

445 (Carl Zeiss Microscopy GmbH, Goettingen, Germany). Images were analysed with Zeiss

446 ZEN2Lite.

447

\section{Behavioral analysis of 4 dpf and 5 dpf ca6 knockdown zebrafish larvae}

Larvae were tested for behavioral consequences due to ca6 knockdown by measuring swimming pattern at $4 \mathrm{dpf}$ and $5 \mathrm{dpf}$. The ca6 knockdown larvae and two controls, namely uninjected wild type and random control MO-injected, were raised in embryo medium. Larvae (approximately 10/flask) were placed in a 23 x 43 x 45 mm TC Flask T25 (Sarstedt AG \& Co, Nümbrecht, Germany) containing $40 \mathrm{ml}$ embryo medium at $3 \mathrm{dpf}$ and allowed to acclimate to the flask for 24 $\mathrm{h}$ at $28.5^{\circ} \mathrm{C}$ standard conditions. At $4 \mathrm{dpf}$ and $5 \mathrm{dpf}$ their swimming patterns were observed by a one-minute video recording, with a printed $1 \mathrm{~cm} \mathrm{x} 1 \mathrm{~cm}$ grid behind the flask. In total, the movement patterns of 284 zebrafish were recorded and measured: 41 of $4 \mathrm{dpf}$ WT, 130 of $4 \mathrm{dpf}$ KD, 32 of $5 \mathrm{dpf}$ WT, and 81 of $5 \mathrm{dpf}$ KD. Sample sizes of at least 30 per group were chosen $a$ priori because normality of distributions could not be assumed.

The movements of all of the larvae were analyzed using the MtrackJ plugin (Meijering, Dzyubachyk \& Smal, 2012) within the ImageJ program (Schneider, Rasband \& Eliceiri, 2012). Tracking and recording of fish movements and analysis of movement data were assigned to two separate researchers to avoid biasing the analysis. Distances traveled (cm per 1 minute, for Fig. 3A) and time spent in the upper half of the tank (seconds, out of $60 \mathrm{~s}$, for Fig. 3B) were calculated for each fish, compiled by group, and presented as boxplots using the Matplotlib (Hunter, 2007) Python library. Statistical testing of similarity between each group, using the Kolmogorov-Smirnov two sample test, was performed using the Stats module of the SciPy Python library (van der Walt, Colbert \& Varoquaux, 2011). The two-sample KolmogorovSmirnov test was chosen because it makes no assumption about the distribution of data.

\section{RESULTS}

\section{Non-mammalian CA VI contains an additional pentraxin domain}

We retrieved $78 \mathrm{CA}$ VI protein sequences from 75 non-mammalian species in NCBI GenPept, all of which have the C-terminal PTX domain. The PTX domain in CA VI is less conserved than the CA domain. The multiple sequence alignment of the $78 \mathrm{CA}$ VI sequences (Fig. S1) shows that there are 83 perfectly conserved amino acids within the catalytic domain (within MSA columns 30-288), whereas only 19 amino acids in the PTX domain are perfectly conserved (within MSA columns 355-566). The region between the CA and PTX domains consists of a moderately conserved and gapless region (MSA columns 300-320) flanked by two highly variable regions of flexible length (MSA columns 292-297 and 326-344). The presence of CA and PTX domains in non-mammalian CA VI sequences has also been documented in the Pfam database since many years (Finn et al., 2016), for example in http://pfam.xfam.org/protein/E9QB97_DANRE. 
488

Fig. 1 presents the phylogenetic tree of CAs VI, IX, XII, and XIV, clearly showing that the longer, non-mammalian isoforms (with a PTX domain) are orthologs of mammalian CA VI. The pairwise arrangement of VI/IX vs. XII/XIV is the same as in previous phylogenetic work (Hewett-Emmett, 2000), suggesting that these four CA isozymes descend from one common ancestor. Fig. 2 shows a phylogenetic tree of all human pentraxins and selected CA-linked PTX domains, which indicates that the novel PTX domains would be most closely related to the short pentraxins, C-reactive protein (CRP) and serum amyloid protein P (APCS or SAP).

\section{Platypus (Ornithorhynchus anatinus) is probably an exception in the pattern of mammals not having a PTX domain associated with CA VI}

A genomic fragment not assigned to any chromosome (Contig22468 in assembly WUGSC 5.0.1/ornAna1) contains an exon which codes for a PTX domain unlike any that we find in other mammalian species, and most similar to CA VI-linked PTX domains in non-mammalian species. The phylogenetic tree in Fig. 2 demonstrates that this platypus PTX sequence is orthologous with the PTX sequences associated with CA VI in non-mammalian species. What is more, a BLASTN search of Contig22468 against the platypus genome showed that it partially matches a region in chromosome 5 right after the CA6 locus. More specifically, the first $703 \mathrm{bp}$ of Contig22468 match the last 703 bases (99.86\% identity, a single mismatch) of Contig3933.5.

The adjacent location of Contig3933.5 to Contig3933.4, the fragment containing the exons coding for the CA6 ortholog (ENSOANG00000013215), would put the exon coding for the PTX domain in the correct location and orientation to be part of the platypus CA6 gene if Contig22468 were placed in this position. Therefore, we tentatively label this PTX domain as "CA-linked" and suggest that Contig22468 would be more correctly mapped starting from Chr5:18954728 in platypus genome assembly OANA5. With this evidence, we also suggest that CA VI in platypus contains a PTX domain, and consequently, that the loss of PTX domain occurred after the separation of monotreme and therian lineages in mammals.

One further phylogenetic tree was made based on CA domain sequences, showing that phylogeny of CA VI follows the expected vertebrate phylogeny, with platypus placed outside of marsupials and placental mammals (Fig. S2).

\section{Exon lengths suggest that the region after the CA domain in CA VI descends from the transmembrane helix of the ancestral form}

Mammalian CA VI proteins contain an additional C-terminal region of at least 25 residues, which is dissimilar to anything in other vertebrate CA isoforms and of unknown structure. Nonmammalian CA VI contains a sequence homologous to this extension as a spacer region between the CA and PTX domains. In order to investigate the most likely origin of the spacer region, we compared the exon lengths in $C A 6$ and the most closely related CA genes $(C A 9, C A 12$, and $C A 14$ ) and short pentraxins. The length of the exon coding for the spacer between CA and PTX domains in zebrafish ca6 is $84 \mathrm{bp}$, and the coding sequence of the homologous exon in human $C A 6$ is $83 \mathrm{bp}$. The exons coding for the region containing the transmembrane (TM) helices (penultimate exons) in $C A 9, C A 12$, and $C A 14$ are $82 \mathrm{bp}, 85 \mathrm{bp}$, and $85 \mathrm{bp}$ in length, respectively. Assuming a novel juxtaposition of exons between genes coding for the ancestral TM form of 
$534 C A 6$ and a short pentraxin, the final exon of $C A 6$ and the first exon of the pentraxin gene are less 535 likely to have been retained. Because they contain non-coding UTR sequences and lack splice 536 donor and acceptor sites, they would be unlikely to be spliced correctly as continuous, protein537 coding sequence. Taken together, this suggests that only the exon coding for the cytoplasmic 538 domain of ancestral CA VI was lost and replaced by the single exon coding for the PTX domain. 539 This also implies that the last exon in mammalian CA6 and the penultimate exon of non-

540 mammalian $C A 6$, predicted to code for an amphipathic helix (see below), and the penultimate 541 exons of $C A 9, C A 12$, and $C A 14$, coding for the transmembrane helix, are highly likely to share a 542 common ancestry.

\section{The region after the $\mathrm{CA}$ domain is predicted to contain an amphipathic helix}

The pattern of hydrophobic residues repeating approximately every fourth residue is obvious in the alignment of the region following the CA domain (final domain in mammalian CA VI, or the segment between CA and PTX domains in non-mammalian CA VI), as seen in Fig. 4C and in the larger alignment of Fig. S1. The helical wheel visualizations of Fig. 4A and 4B indicate that when folded as an alpha helix, this region of human and zebrafish CA VI, respectively, would be an amphipathic helix, with one side lined with mainly hydrophobic residues (in blue and lilac). Furthermore, this region (292 to 312) in zebrafish CA VI is also predicted to have a high potential to form a coiled-coil structure by the COILS algorithm (Lupas, Van Dyke \& Stock, 1991) in InterProScan at http://www.ebi.ac.uk/interpro/sequence-search (Jones et al., 2014). The $\mathrm{APH}$ region is a unique feature of CA VI, present in both non-mammalian and mammalian sequences.

\section{Duplication of an adjacent glucose transporter gene is associated with the loss of PTX from CA VI}

The genes next to $C A 6$ provide a clue for a possible cause of losing the PTX-encoding exon in mammalian $C A 6$. We have observed 17 non-mammalian genomes with a chromosomal arrangement of $C A 6$, then one glucose transporter gene (SLC2A5/SLC2A7), followed by the gene GPR157, whereas most mammalian genomes present the gene order CA6, SLC2A7, SLC2A5, and GPR 157. The reconstructed syntenic block for therian mammals in the region after $C A 6$ in Genomicus (http://www.genomicus.biologie.ens.fr/genomicus-86.01) (Muffato et al., 2010) also shows the duplicated glucose transporter, whereas those for ancestral tetrapods and bony fish lineages only have a single $S L C 2 A 5 / S L C 2 A 7$ ortholog. We were not able to find any single genome containing a PTX-coding exon with $C A 6$ and both $S L C 2 A 5$ and $S L C 2 A 7$. Hence, the available genomic evidence suggests that the loss of the PTX-domain-coding exon and the duplication of the adjacent glucose transporter gene may have occurred simultaneously, close to the divergence time of the mammalian lineage. The rearrangements during the gene duplication would also provide a plausible mechanism for the exon loss.

\section{Sequencing of zebrafish ca6 cDNA confirms a 530-residue product}

We produced a PCR-amplified cDNA of zebrafish ca6 for recombinant protein production. The resulting sequence had five synonymous substitutions compared to Ensembl 
580 ENSDART00000132733 (Fig. S4) and three unresolved bases leading to one unknown amino 581 acid residue. Except for the unknown residue, the translation is identical to the predicted 530582 residue protein (Ensembl ENSDARP00000119189 or UniProt E9QB97, Fig. S5). The cDNA 583 sequence has been submitted to ENA database (http://www.ebi.ac.uk/ena) as LT724251 and its 584 translation to UniProt as A0A1R4AHH7. The other predicted Ensembl transcript 585 (ENSDART00000079007) codes for a protein of 538 residues, in which an additional 24-bp 586 exon creates an insertion before the PTX domain.

587

\section{Sequence alignment predicts three disulfides in zebrafish CA VI}

Cysteine pairs 44/226 in (CA domain, MSA columns 51/234 in Fig. S1), 352 /408, and 487/518 (PTX domain, columns 390/453 and columns 532/564, respectively in Fig. S1) are expected to form disulfides by sequence conservation in the multiple sequence alignment. All three disulfides are also structurally verified. The one in CA domain is seen in all structures of extracellular CAs, e.g. human CA VI in PDB 3FE4 (Pilka et al., 2012), and the disulfide 352/408 in the PTX domain is homologous to the one in short pentraxins, e.g. human C-reactive protein in PDB 3PVN (Guillon et al., 2014). The third disulfide, 487/518, is also supported by proximity in our molecular model (Fig. 5A). There is one further unpaired Cys290, in the region between the CA and PTX domains (and missing from the model), which is also conserved in 76 of 78 non-mammalian sequences (Fig. S1).

\section{D model of zebrafish CA VI-PTX is compatible with predicted amphipathic helix and disulfides}

We made a homology-based model of the CA and PTX domains and combined it with an alpha helical model of the predicted APH region, using protein-protein docking to create the nearly full model. Fig. 5A shows the model, in two orientations, with the CA domain at the top and the PTX domain at the bottom. The APH (pink) fills a non-polar cavity on the surface of the CA domain. The precise orientation of the PTX domain is impossible to predict with certainty, but the current model shows it leaning against the CA domain and APH. The most highly variable regions, for which no template was available, were not modelled (residues 281 to 292 and 311 to 317), indicated by yellow dotted lines (Fig. 5A). In addition, the N-terminus of the model is incomplete, missing residues 20 to 31, which are not visible in the template 3FE4.

The zinc-binding histidines in the active site of the CA domain are shown as yellow sticks in Fig. 5A (zinc not shown), with the active-site cavity opening upwards. Disulfide-forming cysteines are presented as orange stick models. The disulfide in the CA domain and the one in the beta sheet of the PTX domain (lowest in Fig. 4A) are also present in the templates. The information of the predicted third disulfide on the surface of the PTX domain was not used when building the model, but the cysteines ended in close proximity so that the disulfide could be constructed by minor refinement of the model. This disulfide would lock the C-terminus of the PTX domain on the surface of the domain. The presumably unpaired Cys 290 is part of an unmodelled region.

Based on the pentamerization tendency of mammalian PTX domains, we constructed an additional pentameric model of zebrafish CA VI (Fig. 5B-D) by superimposing the PTX domains of five copies of the monomer model on the pentameric structure of SAP (PDB 4AVS). 
626 Individual monomers are presented in different surface colors. There are no serious steric clashes 627 in the model, and the domain axes align to make a flat pentamer complex (Fig. 5D), even if no 628 pentamer constraints were applied for the monomer model. Furthermore, adjacent monomers 629 form an additional protein-protein interface between the sides of their PTX and CA domains. 630 The general shape of the modelled pentamer is a flat, roughly planar five-pointed star, thickness 4 to $5 \mathrm{~nm}$ and an approximate diameter $15 \mathrm{~nm}$. The active site of CA faces outward in the pentamer so that the zinc-binding histidines (yellow spheres in panels B to D) are exposed in the active-site cavity, as seen in the center of panel D.

The four potentially N-glycosylated Asn residues (in the motif Asn-X-Ser/Thr) are all on the surface of the monomer, shown as spheres in Fig. 4A. In contrast, the pentamer model only shows three of them on the surface of the pentamer. Asn210, shown in cyan, is buried between the monomers, conforming well with the observed non-glycosylated status for this Asn residue. The coloring of the potential glycosylation sites in Figs. 4A-D reflects their observed glycosylation status (presented below under mass spectrometry).

\section{Recombinant CA VI-PTX shows a high catalytic activity}

Zebrafish CA VI-PTX was produced in insect cells with high yield. The purified protein showed a single band close to the expected size in SDS-PAGE (Fig. 6, measured molecular weight 58.6 $\mathrm{kDa}$, theoretical $58.107 \mathrm{kDa}$ without glycans, signal peptide excluded). Carbonate dehydratase activity was analyzed kinetically in the presence or absence of acetazolamide. The kinetic parameters of CA VI-PTX $\left(\mathrm{k}_{\mathrm{cat}}\right.$ and $\left.\mathrm{k}_{\mathrm{cat}} / \mathrm{K}_{\mathrm{m}}\right)$ were then compared with those of the thoroughly investigated CAs, namely the cytosolic and ubiquitous human isozymes $\alpha$-CA I (hCA I) and II (hCA II). The CA VI-PTX possesses considerable carbonate dehydratase activity as shown in Table 2. A $\mathrm{k}_{\text {cat }}$ of $8.9 \times 10^{5} \mathrm{~s}^{-1}$ and a $\mathrm{k}_{\text {cat }} / \mathrm{K}_{\mathrm{m}}$ of $1.3 \times 10^{8} \mathrm{M}^{-1} \mathrm{x} \mathrm{s}^{-1}$ show that the enzymatic activity of CA VI-PTX is almost in the same range with the very highly active human CA II. Data also show that CA VI-PTX was efficiently inhibited, with an inhibition constant of $5 \mathrm{nM}$, by the clinically-used sulfonamide, acetazolamide (5-acetamido-1,3,4-thiadiazole-2-sulfonamide).

\section{Light scattering analysis by LC-SLS-DLS confirms multimeric structure}

The molecular size of native recombinantly produced zebrafish CA VI-PTX was estimated by static and dynamic light scattering (SLS and DLS) analysis after liquid chromatography. Gel filtration analysis indicated main peak eluting at $1.52 \mathrm{ml}$ retention volume according to A280

661 (Fig. 7, black curve). This was associated with static light scattering intensity peak with identical shape. Analysis of the scattering intensity (SLS) results in a MW estimate of $280 \pm 11 \mathrm{kDa}$ for the peak, and the estimate was homogeneous throughout the elution peak (Fig. 7, near-horizontal line across the peak in dark gray). In addition, DLS data was collected for the eluted peak indicating particle size of $7.69 \pm 0.29 \mathrm{~nm}$, as $\mathrm{Rh}$ (hydrodynamic radius), which is consistent with the determined molecular weight. The MW estimate based on the retention volume in gel filtration is slightly smaller $(214 \pm 10 \mathrm{kDa})$, possibly due to off-globular shape of the molecule. The small peak eluting before the main peak $(\sim 1.1 \mathrm{ml}$ retention volume $)$ indicated the presence of aggregated protein, resulting in high scattering intensity. According to A280, this is less than $5 \%$ of the protein sample. Altogether, the light scattering analysis combined with gel filtration 
671 indicates oligomeric assembly for the protein, a pentameric form being the most probable 672 oligomeric state.

673

674

675

676

677

678

679

680

681

682

683

684

685

686

687

688

689

690

691

692

693

694

695

696

697

698

699

700

701

702

703

704

705

706

707

708

709

710

711

712

713

714

715

716

\section{Mass spectrometry confirms post-translational modifications}

All attempts to characterize the intact CA VI-PTX with ESI FT-ICR mass spectrometry failed, despite the extensive sample desalting/purification prior to the measurements. This may be due to a slight protein precipitation observed during the sample preparation. Therefore, in-solution trypsin digestion was selected as the main route for structural characterization of CA VI-PTX. The digestion was performed in non-reducing conditions to preserve disulfide bonds in the structure. The digestion resulted in $97 \%$ sequence coverage with 64 specific tryptic peptides identified (Figs. 8 and 9, and fuller details in Fig. S6 and Table S1).

The peptide map in Fig. 9 shows that the tryptic peptides were found within both protein domains, although somewhat larger peptides (up to $\sim 14 \mathrm{kDa}$ ) were found within the PTX domain. Out of all identified peptides twelve contained disulfide bonds (either intra- or interpeptide). These peptides confirmed the putative disulfide bonds, Cys 44/226 in the CA domain, and Cys 352/408 and Cys 487/518 in the PTX domain. Cys290 in the spacer region is most likely free but the corresponding tryptic peptide (LSKGGMCR) was not observed to confirm this. These disulfide bonds are fully consistent with the 3D structural model of CA VIPTX.

CA VI-PTX contains four putative N-glycosylation sites (Asn210, Asn258, Asn339 and Asn394), having a canonical NxS/T consensus sequence (marked in Fig. 9). Among the identified tryptic peptides, twelve glycopeptides were found. On the basis of these peptides, CA VI-PTX carries two glycans, a core-fucosylated oligomannose type glycan $\mathrm{GlcNAc}_{2}(\mathrm{Fuc}) \mathrm{Man}_{3}$ at Asn258 and an oligomannose type glycan GlcNAc ${ }_{2} \mathrm{Man}_{3}$ at Asn339, located in the CA domain and PTX domain, respectively. These glycosylation sites and glycan structures were further verified by CID-MS/MS experiments of the representing glycopeptides [248-266] (3416.5084 Da) and [331-347] (2819.3059 Da) (Fig. 10). As no other glycan variants were observed among the peptides, it seems that the glycosylation in CA VI-PTX (produced in insect cells) is rather homogenous. These results are consistent with accessibility of the sites predicted by our model. Interestingly, the glycosylation site at Asn 258 is conserved in 77 out of 78 non-mammalian CA VI sequences in the sequence alignment Fig. S1 (columns 266 - 268). The tryptic peptide [191216] (2988.4744 Da) was only observed in a free form, indicating that Asn 210 is nonglycosylated in the CA domain. Similarly, the peptides spanning the Asn394 residue were all observed without any glycans attached (Fig. S6), suggesting that this site is non-glycosylated in the PTX domain.

\section{Immunohistochemistry shows cell surface localization of CA VI-PTX in various tissues}

Recombinant zebrafish CA VI-PTX protein was used to raise a rabbit polyclonal antiserum, which worked well in immunofluorescence studies. Fig. 11 shows positive staining in the skin, heart, gills, and swim bladder. The strongest signal is seen on cell surfaces, while the intracellular staining was detectable but weaker. 
717 To get further insights into ca6 expression in zebrafish, we also studied the expression pattern in 718 different tissues of adult zebrafish by qRT-PCR. As shown in Table 3, relative expression of ca6 719 mRNA was found to be prominent in the fins/tail, and brain. Low levels of expression were 720 observed in the gills, kidney, teeth, skin, and spleen. A very faint signal was detected in the swim

\section{Zebrafish cannot swim properly in the ca6 knockdown model}

Gene-specific antisense morpholino oligonucleotides have been widely used to inhibit gene expression in zebrafish larvae (Eisen, Smith, 2008). We designed two different MOs, one for translational blocking of ca6 mRNA and the other for blocking intron splicing before exon 9 . Both MOs were used to repeat all knockdown experiments with highly similar results, suggesting equally efficient knockdown in both kinds of ca6 morphants. We did not see any morphological differences between uninjected and random control MO-injected embryos/larvae over the period of 5 days of development. The ca6 morphant zebrafish embryos between 1 and $3 \mathrm{dpf}$ were also devoid of any notable morphological changes, but interestingly, at the end of $4 \mathrm{dpf}$ we consistently observed an underdeveloped or deflated swim bladder in ca6 morphant larvae (Fig. 12).

The quantitative expression analysis of ca6 mRNA was done in wild-type and the ca6 morphant zebrafish at different stages of development. As seen in Fig. 13, the mRNA expression in wildtype embryos was highest at $24 \mathrm{hpf}$, with slightly lower values later. The levels of ca6 mRNA were consistently higher in the morphant embryos compared to the wild type, possibly because of compensatory upregulation of the gene caused by the absence of CA VI protein. The peak expression of ca6 was at $48 \mathrm{hpf}$ in the ca6 morphant embryos.

In order to measure swimming activity of morphant vs. wild type, we calculated total distances traveled for individual larvae, and they are presented as boxplots in Fig. 3. Two-sample Kolmogorov-Smirnov statistical analyses were performed between relevant group pairs to determine if they could have been drawn from the same distribution. Day 4 knockdown larvae swam less (median $0.00 \mathrm{~cm}$ ) than day 4 wild-type larvae (median $13.80 \mathrm{~cm}$, p-value $4.28 \times 10^{-}$ ${ }^{19}$ ), and similarly day 5 knockdown larvae swam less (median $4.75 \mathrm{~cm}$ ) than day 5 wild-type larvae (median $10.22 \mathrm{~cm}$, p-value $1.16 \times 10^{7}$ ). Full details of the swimming data are shown in Table 4. Taken together with the clearly observed swim bladder deficiency in 4 dpf larvae (Fig. 12) and the presence of CA VI in adult zebrafish swim bladder, we suggest that CA VI is required either for swim bladder development or swim bladder function. When CA VI expression is mainly restored in $5 \mathrm{dpf}$ larvae, the swimming pattern also returns to almost normal.

\section{DISCUSSION}

This study consists of the characterization of a novel type of a carbonic anhydrase, CA VI containing a pentraxin domain, by means of sequence analyses, phylogenetics, molecular modelling, experiments on a recombinantly produced protein, knockdown of the ca6 gene in zebrafish embryos, and expression studies by immunohistochemistry and qRT-PCR. The bioinformatic and experimental analyses build a coherent picture of the structure of this novel 
763

764

765

766

767

domain combination, and the evolutionary analysis shows a history of domain gains and losses. Based on our previous work and the findings in this study, we propose that CA VI-PTX in zebrafish is needed for filling the swim bladder, and possibly in a novel type of membrane anchoring and immune function.

The pentraxin domain found associated with non-mammalian CA VI is a novel member of the pentraxin family. We have shown it to be most closely related with the short pentraxins, CRP and SAP (Fig. 2). The association of a CA domain with a PTX domain is new in both the pentraxin and carbonic anhydrase families. SAP and CRP are more closely similar to each other than either is to the CA-associated PTX domain. This could indicate that the CA-associated PTX domain had diverged from a common ancestor before the duplication that created SAP and CRP, but we cannot take this for granted, because adaptation to create a viable domain interface may have accelerated the rate of change in the CA-associated PTX domain.

The phylogenetic tree in Fig. 1 shows that the transmembrane CAs IX, XII, and XIV and secretory CA VI share a common ancestor. We propose that the quartet has arisen in the two whole-genome duplications in early vertebrates. Fig. 14 presents a plausible sequence of events that could have led to present-day domain structures in CA VI. Briefly, we assume that the exon coding for the cytoplasmic domain in ancestral CA VI was replaced by an exon coding for a PTX domain (probably by a duplication or a move of an exon coding for a short pentraxin in early vertebrates), and the TM helix transformed into an amphipathic helix (Figs. 4 and 5). Later, presumably in the therian mammal lineage, the PTX domain was lost, leaving the APH in the Cterminus of CA VI. These hypotheses are supported by the following observations: 1) Comparison of exon lengths suggests the TM-helix-coding exon as the most likely ancestor of the exon coding the spacer region after the CA domain in CA VI; 2) the losses of the CP domain in early CA VI and of PTX domain in mammalian lineage are more parsimonious assumptions than their acquisition in multiple lineages; 3 ) the duplication of the glucose transporter genes $S L C 2 A 5$ and $S L C 2 A 7$, as seen in therian mammals, is evidence of rearrangements in the region adjacent to the PTX-domain-coding exon of the $C A 6$ locus, which we assume to have led to the loss of the PTX domain in mammalian CA VI; and 4) the PTX domain is consistently present in non-mammalian CA VI and missing from mammalian CA VI (most likely excepting platypus).

Considering the monomer MW of $58.1 \mathrm{kDa}$ (plus glycosylation), the LC-SLS-DLS results clearly confirm that zebrafish CA VI is an oligomer. The MW estimated by LC- SLS (280 \pm 11 $\mathrm{kDa})$ is slightly less than MW calculated from sequence $(290.5 \mathrm{kDa}$ for pentamer, plus glycosylation). Based on the gel filtration retention volume and protein standards, the MW is estimated to be slightly smaller $(214 \pm 10 \mathrm{kDa})$, but this result may be affected by column interactions and deviation from the globular shape. Furthermore, the hydrodynamic radius calculated from light scattering $(7.69 \pm 0.29 \mathrm{~nm}$; diameter $15.38 \pm 0.58 \mathrm{~nm})$ suggests a particle size in the range of 364 to $434 \mathrm{kDa}$ for globular particle. In this context, it has to be noted that diffusion of the particle is highly dependent on the molecular shape and DLS-based estimate may also be slightly affected by irregular shape. Taken together, the light scattering results are more compatible with a pentamer than tetramer or hexamer models. The 3D model of CA VI-PTX as a pentamer (Fig. 5B-D) predicts a shape of a flat, roughly planar five-pointed star, thickness 4 to 5 $\mathrm{nm}$ and approximate diameter $15 \mathrm{~nm}$, i.e. clearly off-globular, which would explain the minor conflicts between observations. What is more, the pentamer model is also supported by known 
809 pentamerization of related pentraxins (CRP and SAP). However, we need to stress that the

810

811

812

813

814

815

816

817

818

819

820

821

822

823

824

825

826

827

828

829

830

831

832

833

834

835

836

837

838

839

840

841

842

843

844

845

846

847

848

849

850

851

852

853 relative orientations of the CA and PTX domains in our models are only tentative.

Mass spectrometry confirms that the N-terminus of the mature CA VI-PTX coincides with the predicted signal peptide cleavage site between residues 19 and 20. Glycopeptides with typical Nlinked glycans are observed associated with Asn258 and Asn339, whereas the peptides containing Asn 210 or Asn394 are only seen in non-glycosylated form (Fig. 9). Consistent with these observations of N-glycosylation, our 3D model of pentameric CA VI-PTX (Fig. 5B-D) shows that Asn258 and Asn339 are well exposed, whereas Asn210 is fully buried in the protomer/domain interface, and Asn394 would be somewhat hindered at the protomer interface.

We discovered a minor but surprising outcome in the knockdown model regarding the poor floating ability, most likely caused by a deflated swim bladder, both of which we observed consistently in $4 \mathrm{dpf}$ knockdown larvae. The statistically significant lower swimming distances and stationary positioning at the bottom of $4 \mathrm{dpf}$ knockdown larvae, versus those of $4 \mathrm{dpf}$ wildtype larvae or $5 \mathrm{dpf}$ knockdown larvae, imply that the knockdown larvae gain normal swimming function as the knockdown action of the injected morpholino oligonucleotides is relieved (Fig. 12). CA VI-PTX function within the swim bladder is further supported by immunohistochemistry and qRT-PCR, showing expression of both CA VI-PTX protein and mRNA in the swim bladder specimens. However, at the current point we cannot distinguish whether the swim bladder dysfunction observed in $4 \mathrm{dpf}$ larvae is due to delayed development or the need of CA VI-PTX in swim bladder inflation.

CRP and SAP are known to bind carbohydrates, i.e. they are lectins (Hind et al., 1984; Kottgen et al., 1992). The calcium-binding residues in the sugar binding site are partially conserved between these two lectins and the CA-associated PTX domain. In addition, pentraxins are a subfamily of the Concanavalin A-like lectin/glucanase family, which contains numerous other lectins (leguminous plant lectins, animal galectins, etc.) and other proteins interacting with carbohydrates (http://www.ebi.ac.uk/interpro/entry/IPR013320). In our immunohistochemistry results the CA VI-PTX protein shows mostly a strong cell-surface staining pattern (Fig. 11), even if the protein is predicted to be a secreted, soluble protein. We assume that the PTX domain in CA VI would also be a lectin and anchor the protein on the cell surface via sugars in glycoconjugates. Binding to plasma membrane glycoconjugates would also explain why the loss of the TMH was tolerated, i.e. transmembrane helix anchoring was replaced by lectin anchoring. If sugar binding by CA VI-PTX can be proved experimentally, non-mammalian CA VI would represent the first case of an enzyme which is attached on the cell surface by lectin binding.

Lectins and other pattern-recognition molecules are an important part of the innate immune system in fishes, which is more diverse than that of mammals (Vasta et al., 2011; Sunyer, Zarkadis \& Lambris, 1998). Although teleost fish lack lymph nodes and bone marrow, the anterior part of the fish kidney is considered a functional ortholog of mammalian bone marrow. Thus, it represents the main hematopoietic lymphoid tissue of teleosts, and is thought to be an immunologically responsive organ (Zapata, Amemiya, 2000). The role of maintenance of mucosal homeostasis is served in teleosts by the gut, skin, and gills, which all contain mucosaassociated lymphoid tissue (Salinas, Zhang \& Sunyer, 2011). These are among the tissues where 
854

855

856

857

858

859

860

861

862

863

864

865

866

867

868

869

870

871

872

873

874

875

876

877

878

879

880

881

882

883

884

885

886

887

888

889

890

891

892

893

894

895

896

zebrafish CA VI-PTX has its highest expression, and therefore we assume that this protein is a part of the innate immune system.

Interestingly, we have shown that mouse CA VI is also highly expressed in the gut, specifically in the immunologically active Peyer's patches (Pan et al., 2011). In another study, we demonstrated that there is a likely role for Car6 in immune stimulated lung tissues (Patrikainen et al., 2016) and murine Car6 is likely involved in mucosa maintenance in both airways and gut (Leinonen et al., 2004; Parkkila et al., 1997). We formed a preliminary hypothesis that mouse CA VI is involved in immunological functions, which has been confirmed recently (Xu et al., 2017), by showing that CA VI isoform B promotes interleukin-12 expression. However, a gene regulatory function is unlikely for zebrafish CA VI, with the estimated diameter of $15 \mathrm{~nm}$ for the pentamer making it too large to enter the nuclear pores. The locations of high ca6/CA6 expression in fish and in mammals are similar in that they allow delivery of CA VI on the physical barrier against external environment (gut, skin, and gills in zebrafish; skin, saliva, milk, and lungs in human/mouse), consistent with a function associated with primary immune defense. Summing up, we suggest that both mammalian and fish CA VI are components of the innate immune system, with or without a PTX domain.

Given the dynamic nature of genomes, with transposition and translocation events constantly shuffling exons, it is hard to see the choreography of domain moves in CA VI during vertebrate evolution as anything more than chance events. However, in order to remain stably in a genome, the changes must be at least tolerated, or possibly provide some advantage to their carrier. We see the addition of the PTX domain in early jawed vertebrates as a tolerated change, in which membrane attachment through a transmembrane helix was replaced by lectin anchoring. As we have suggested, the new domain context may have led to the CA domain of CA VI adopting functionality within the innate immune system. Then later, when the PTX domain was lost, presumably through the local segmental duplication leading to a duo of glucose transporters (SLC2A5 and SLC2A7), the addition of another glucose transporter may have been more of an advantage than the loss of pentamerization and membrane anchoring in CA VI, and thus this chromosomal arrangement became fixed in early therian mammals. The loss of the PTX domain may also have opened the way for using the amphipathic helix in forming dimers. We have a preliminary result of human CA VI being a mixture of monomer and dimer forms in solution (A. Yrjänäinen, unpublished), in which we speculate dimerization to be mediated by the amphipathic helices being able to join in a coiled-coil fashion when unhindered by a further C-terminal domain.

This study has given us many ideas for future research. We plan to take a closer look at the complex evolution of non-mammalian pentraxins, which might shed more light on the origin of the CA VI-linked PTX domain and on structure-related constraints on its surface. We have also started work on comparisons of per-residue conservation patterns of the CA domain in mammalian vs. non-mammalian CA VI. Testing the sugar-binding ability of CA VI-PTX will be the obvious way to explore the lectin hypothesis. 
897

898

899

900

901

902

903

904

905

906

907

908

909

910

911

912

913

914

915

916

917

918

919

920

921

922

923

924

925

926

927

928

929

930

931

932

933

934

935

\section{Data availability}

The zebrafish ca6 cDNA sequence has been deposited to the ENA database (http://www.ebi.ac.uk/ena) and assigned the identifier LT724251. The translated protein sequence is available in UniProt (http://www.uniprot.org) as A0A1R4AHH7.

\section{Acknowledgments}

We thank Aulikki Lehmus and Marianne Kuuslahti for the skillful technical assistance with most experiments; Leena Mäkinen, and Hannaleena Piippo, for their technical assistance with zebrafish experiments, and Jukka Lehtiniemi for the artwork of Fig. 14. Thanks are due to Alma Yrjänäinen and Linda Urbański for the help with immunohistochemistry experiments and collecting tissues. We thank Mataleena Parikka for the help with adult zebrafish tissue collection. The authors thank Ritva Romppanen for preparing samples for mass spectrometry analysis. We acknowledge Biocenter Finland for infrastructure support in light scattering experiments. Core facilities at BioMediTech and Faculty of Medicine and Life Sciences, University of Tampere, were essential in microscopy (Tampere Imaging Facility), zebrafish experiments (Zebrafish Laboratory), and in DNA sequencing (Sequencing Facility).

\section{REFERENCES}

Altschul SF, Gish W, Miller W, Myers EW, Lipman DJ. 1990. Basic local alignment search tool. Journal of Molecular Biology, 215:403-410.

Berrino E, Bua S, Mori M, Botta M, Murthy VS, Vijayakumar V, Tamboli Y, Bartolucci G, Mugelli A, Cerbai E, Supuran CT, Carta F. 2017. Novel Sulfamide-Containing Compounds as Selective Carbonic Anhydrase I Inhibitors. Molecules (Basel, Switzerland), 22:10.3390/molecules22071049.

Bottazzi B, Inforzato A, Messa M, Barbagallo M, Magrini E, Garlanda C, Mantovani A. 2016. The pentraxins PTX3 and SAP in innate immunity, regulation of inflammation and tissue remodelling. Journal of hepatology, 64:1416-1427.

de Vries SJ, Bonvin AM. 2011. CPORT: a consensus interface predictor and its performance in prediction-driven docking with HADDOCK. PloS one, 6:e17695.

de Vries SJ, van Dijk AD, Krzeminski M, van Dijk M, Thureau A, Hsu V, Wassenaar T, Bonvin AM. 2007. HADDOCK versus HADDOCK: new features and performance of HADDOCK2.0 on the CAPRI targets. Proteins, 69:726-733.

Eisen JS, Smith JC. 2008. Controlling morpholino experiments: don't stop making antisense. Development (Cambridge, England), 135:1735-1743.

Fernley RT, Wright RD, Coghlan JP. 1979. A novel carbonic anhydrase from the ovine parotid gland. FEBS letters, 105:299-302. 
936 Finn RD, Coggill P, Eberhardt RY, Eddy SR, Mistry J, Mitchell AL, Potter SC, Punta M, 937 Qureshi M, Sangrador-Vegas A, Salazar GA, Tate J, Bateman A. 2016. The Pfam protein 938 families database: towards a more sustainable future. Nucleic acids research, 44:D279-85.

939 Flicek P, Amode MR, Barrell D, Beal K, Brent S, Carvalho-Silva D, Clapham P, Coates G, 940 Fairley S, Fitzgerald S, Gil L, Gordon L, Hendrix M, Hourlier T, Johnson N, Kahari AK, Keefe 941 D, Keenan S, Kinsella R, Komorowska M, Koscielny G, Kulesha E, Larsson P, Longden I, 942 McLaren W, Muffato M, Overduin B, Pignatelli M, Pritchard B, Riat HS, Ritchie GR, Ruffier 943 M, Schuster M, Sobral D, Tang YA, Taylor K, Trevanion S, Vandrovcova J, White S, Wilson M, 944 Wilder SP, Aken BL, Birney E, Cunningham F, Dunham I, Durbin R, Fernandez-Suarez XM, 945 Harrow J, Herrero J, Hubbard TJ, Parker A, Proctor G, Spudich G, Vogel J, Yates A, Zadissa A, 946 Searle SM. 2012. Ensembl 2012. Nucleic acids research, 40:D84-90.

947 Garlanda C, Bottazzi B, Bastone A, Mantovani A. 2005. Pentraxins at the crossroads between 948 innate immunity, inflammation, matrix deposition, and female fertility. Annual Review of 949 Immunology, 23:337-366.

950 Gasteiger E, Gattiker A, Hoogland C, Ivanyi I, Appel RD, Bairoch A. 2003. ExPASy: The 951 proteomics server for in-depth protein knowledge and analysis. Nucleic acids research, 31:37849523788.

953 Guillon C, Bigouagou UM, Folio C, Jeannin P, Delneste Y, Gouet P. 2014. A staggered 954 decameric assembly of human C-reactive protein stabilized by zinc ions revealed by X-ray 955 crystallography. Protein and Peptide Letters, 22:248-255.

956

957

958

959

960

961

962

963

964

965

966

967

968

969

970

Henkin RI, Lippoldt RE, Bilstad J, Edelhoch H. 1975. A zinc protein isolated from human parotid saliva. Proceedings of the National Academy of Sciences, 72:488-492.

Hewett-Emmett D. 2000. Evolution and distribution of the carbonic anhydrase gene families. EXS, (90):29-76.

Hind CR, Collins PM, Renn D, Cook RB, Caspi D, Baltz ML, Pepys MB. 1984. Binding specificity of serum amyloid $\mathrm{P}$ component for the pyruvate acetal of galactose. The Journal of experimental medicine, 159:1058-1069.

Hunter JD. 2007. Matplotlib: A 2D Graphics Environment. Computing in Science Engineering, 9:90-95.

Jones P, Binns D, Chang HY, Fraser M, Li W, McAnulla C, McWilliam H, Maslen J, Mitchell A, Nuka G, Pesseat S, Quinn AF, Sangrador-Vegas A, Scheremetjew M, Yong SY, Lopez R, Hunter S. 2014. InterProScan 5: genome-scale protein function classification. Bioinformatics (Oxford, England), 30:1236-1240.

Karhumaa P, Leinonen J, Parkkila S, Kaunisto K, Tapanainen J, Rajaniemi H. 2001. The identification of secreted carbonic anhydrase VI as a constitutive glycoprotein of human and rat 
971

972

973

974

975

976

977

978

979

980

981

982

983

984

985

986

987

988

989

990

992

993

994

milk. Proceedings of the National Academy of Sciences of the United States of America, 98:11604-11608.

Khalifah RG. 1971. The carbon dioxide hydration activity of carbonic anhydrase. I. Stop-flow kinetic studies on the native human isoenzymes B and C. The Journal of biological chemistry, 246:2561-2573.

Kolstoe SE, Jenvey MC, Purvis A, Light ME, Thompson D, Hughes P, Pepys MB, Wood SP. 2014. Interaction of serum amyloid P component with hexanoyl bis(D-proline) (CPHPC). Acta crystallographica.Section D, Biological crystallography, 70:2232-2240.

Kottgen E, Hell B, Kage A, Tauber R. 1992. Lectin specificity and binding characteristics of human C-reactive protein. Journal of immunology (Baltimore, Md.: 1950), 149:445-453.

Leinonen JS, Saari KA, Seppanen JM, Myllyla HM, Rajaniemi HJ. 2004. Immunohistochemical demonstration of carbonic anhydrase isoenzyme VI (CA VI) expression in rat lower airways and lung. The journal of histochemistry and cytochemistry : official journal of the Histochemistry Society, 52:1107-1112.

Leinonen J, Parkkila S, Kaunisto K, Koivunen P, Rajaniemi H. 2001. Secretion of Carbonic Anhydrase Isoenzyme VI (CA VI) from Human and Rat Lingual Serous von Ebner's Glands. Journal of Histochemistry \& Cytochemistry, 49:657-662.

Lupas A, Van Dyke M, Stock J. 1991. Predicting coiled coils from protein sequences. Science (New York, N.Y.), 252:1162-1164.

Medzhitov R. 2007. Recognition of microorganisms and activation of the immune response. Nature, 449:819-826.

Meijering E, Dzyubachyk O, Smal I. 2012. Methods for cell and particle tracking. Methods in enzymology, 504:183-200.

Melis M, Atzori E, Cabras S, Zonza A, Calo C, Muroni P, Nieddu M, Padiglia A, Sogos V, Tepper BJ, Tomassini Barbarossa I. 2013. The Gustin (CA6) Gene Polymorphism, rs2274333 $(\mathrm{A} / \mathrm{G})$, as a Mechanistic Link between PROP Tasting and Fungiform Taste Papilla Density and Maintenance. PloS one, 8:e74151.

Muffato M, Louis A, Poisnel CE, Roest Crollius H. 2010. Genomicus: a database and a browser to study gene synteny in modern and ancestral genomes. Bioinformatics (Oxford, England), 26:1119-1121.

NCBI Resource Coordinators. 2016. Database resources of the National Center for Biotechnology Information. Nucleic acids research, 44:D7-19. 
1003 Pan PW, Kayra K, Leinonen J, Nissinen M, Parkkila S, Rajaniemi H. 2011. Gene expression 1004 profiling in the submandibular gland, stomach, and duodenum of CAVI-deficient mice.

1005 Transgenic research, 20:675-698.

1006 Paradis E, Claude J, Strimmer K. 2004. APE: Analyses of Phylogenetics and Evolution in R 1007 language. Bioinformatics (Oxford, England), 20:289-290.

1008 Parkkila S, Parkkila A, Lehtola J, Reinila A, Sodervik H, Rannisto M, Rajaniemi H. 1997. 1009 Salivary carbonic anhydrase protects gastroesophageal mucosa from acid injury. Digestive 1010 diseases and sciences, 42:1013-1019.

1011 Parkkila S, Kaunisto K, Rajaniemi L, Kumpulainen T, Jokinen K, Rajaniemi H. 1990.

1012 Immunohistochemical localization of carbonic anhydrase isoenzymes VI, II, and I in human 1013 parotid and submandibular glands. The journal of histochemistry and cytochemistry : official 1014 journal of the Histochemistry Society, 38:941-947.

1015 Parkkila S, Parkkila AK, Vierjoki T, Stahlberg T, Rajaniemi H. 1993. Competitive time-resolved 1016 immunofluorometric assay for quantifying carbonic anhydrase VI in saliva. Clinical chemistry, 1017 39:2154-2157.

1018 Patrikainen M, Pan P, Kulesskaya N, Voikar V, Parkkila S. 2014. The role of carbonic anhydrase 1019 VI in bitter taste perception: evidence from the Car6(-)/(-) mouse model. Journal of Biomedical 1020 Science, 21:82-014-0082-2.

1021 Patrikainen MS, Pan P, Barker HR, Parkkila S. 2016. Altered gene expression in the lower 1022 respiratory tract of Car6 (-/-) mice. Transgenic research, 25:649-664.

1023

1024

1025

1026 1027

1028

1029

1030

1031

1032

1033

1034

1035

Pettersen EF, Goddard TD, Huang CC, Couch GS, Greenblatt DM, Meng EC, Ferrin TE. 2004. UCSF Chimera--a visualization system for exploratory research and analysis. Journal of computational chemistry, 25:1605-1612.

Pfaffl MW. 2001. A new mathematical model for relative quantification in real-time RT-PCR. Nucleic Acids Res, 29:e45.

Pilka ES, Kochan G, Oppermann U, Yue WW. 2012. Crystal structure of the secretory isozyme of mammalian carbonic anhydrases CA VI: implications for biological assembly and inhibitor development. Biochemical and biophysical research communications, 419:485-489.

Rice P, Longden I, Bleasby A. 2000. EMBOSS: the European Molecular Biology Open Software Suite. Trends in genetics : TIG, 16:276-277.

Ronquist F, Teslenko M, van der Mark P, Ayres DL, Darling A, Hohna S, Larget B, Liu L, Suchard MA, Huelsenbeck JP. 2012. MrBayes 3.2: efficient Bayesian phylogenetic inference and model choice across a large model space. Systematic Biology, 61:539-542. 
1036 Roy A, Kucukural A, Zhang Y. 2010. I-TASSER: a unified platform for automated protein

1037

1038

1039

1040

1041

1042

1043

1044

1045

1046

1047

1048

1049

1050

1051

1052

1053

1054

1055

1056

1057

1058

1059

1060

1061

1062

1063

1064

1065

1066

1067

1068 structure and function prediction. Nature protocols, 5:725-738.

Salinas I, Zhang YA, Sunyer JO. 2011. Mucosal immunoglobulins and B cells of Teleost fish. Developmental and comparative immunology, 35:1346-1365.

Schneider CA, Rasband WS, Eliceiri KW. 2012. NIH Image to ImageJ: 25 years of image analysis. Nature methods, 9:671-675.

Shatzman AR, Henkin RI. 1981. Gustin concentration changes relative to salivary zinc and taste in humans. Proceedings of the National Academy of Sciences, 78:3867-3871.

Sievers F, Wilm A, Dineen D, Gibson TJ, Karplus K, Li W, Lopez R, McWilliam H, Remmert M, Soding J, Thompson JD, Higgins DG. 2011. Fast, scalable generation of high-quality protein multiple sequence alignments using Clustal Omega. Molecular systems biology, 7:539.

Sunyer JO, Zarkadis IK, Lambris JD. 1998. Complement diversity: a mechanism for generating immune diversity?. Immunology today, 19:519-523.

Suyama M, Torrents D, Bork P. 2006. PAL2NAL: robust conversion of protein sequence alignments into the corresponding codon alignments. Nucleic acids research, 34:W609-12.

Thatcher BJ, Doherty AE, Orvisky E, Martin BM, Henkin RI. 1998. Gustin from human parotid saliva is carbonic anhydrase VI. Biochemical and biophysical research communications, 250:635-641.

van der Walt S, Colbert SC, Varoquaux G. 2011. The NumPy Array: A Structure for Efficient Numerical Computation. Computing in Science \& Engineering, 13:22-30.

Vasta GR, Nita-Lazar M, Giomarelli B, Ahmed H, Du S, Cammarata M, Parrinello N, Bianchet MA, Amzel LM. 2011. Structural and functional diversity of the lectin repertoire in teleost fish: relevance to innate and adaptive immunity. Developmental and comparative immunology, 35:1388-1399.

Webb B, Sali A. 2016. Comparative Protein Structure Modeling Using MODELLER. Current protocols in protein science, 86:2.9.1-2.9.37.

Westerfield M. 2007. The zebrafish book: a guide for the laboratory use of zebrafish (Danio rerio). Eugene (OR): M. Westerfield.

Xu J, Xu X, Wang B, Ma Y, Zhang L, Xu H, Hu Y, Wu J, Cao X. 2017. Nuclear carbonic anhydrase 6B associates with PRMT5 to epigenetically promote IL-12 expression in innate response. Proceedings of the National Academy of Sciences, 114:8620-8625.

Zapata A, Amemiya CT. 2000. Phylogeny of lower vertebrates and their immunological structures. Current topics in microbiology and immunology, 248:67-107. 


\section{Table $\mathbf{1}$ (on next page)}

Suggested corrections for predicted translation start sites.

The following database entries have N-terminal extensions which we assume mispredicted. We have shortened these sequences to start at a conserved initiating Met residues for use in this study. 


\section{Table 1. Suggested corrections for predicted translation start sites.}

2 The following database entries have N-terminal extensions which we assume mispredicted, and

3 we have shortened these sequences to start at a conserved initiating Met residues for use in this 4 study.

5

\begin{tabular}{lllc}
\hline RefSeq ID & Name & Organism & $\begin{array}{c}\text { No. of N-terminal } \\
\text { residues removed }\end{array}$ \\
\hline XP_010721064.1 & PREDICTED: carbonic anhydrase 6 & Meleagris gallopavo & 110 \\
XP_005057921.1 & PREDICTED: carbonic anhydrase 6 & Ficedula albicollis & 17 \\
XP_002187446.1 & PREDICTED: carbonic anhydrase 6 & Taeniopygia guttata & 6 \\
XP_005143337.1 & PREDICTED: carbonic anhydrase 6 & Melopsittacus undulatus & 31 \\
\hline
\end{tabular}

6

7

8

9

10

11

12

13

14

15 


\section{Table 2 (on next page)}

Kinetic parameters for $\mathrm{CO}_{2}$ hydration reaction catalysed by selected $\alpha$-CA isozymes. 
2 Table 2. Kinetic parameters for $\mathrm{CO}_{2}$ hydration reaction catalysed by selected $\alpha$-CA

3 isozymes

4

\begin{tabular}{lllll}
\hline Enzyme & $\mathrm{k}_{\mathrm{cat}}\left(\mathrm{s}^{-1}\right)$ & $\mathrm{K}_{\mathrm{m}}(\mathrm{mM})$ & $\mathrm{k}_{\mathrm{cat}} / \mathrm{K}_{\mathrm{m}}\left(\mathrm{M}^{-1} \mathrm{~s}^{-1}\right)$ & $\mathrm{K}_{\mathrm{I}}(\mathrm{AAZ})(\mathrm{nM})$ \\
\hline $\mathrm{hCA} \mathrm{I}^{\mathrm{a}}$ & $2.0 \times 10^{5}$ & 4.0 & $5.0 \times 10^{7}$ & 250 \\
hCA II $^{\mathrm{a}}$ & $1.4 \times 10^{6}$ & 9.3 & $1.5 \times 10^{8}$ & 12 \\
pentraxin-CA VI $^{\mathrm{b}}$ & $8.9 \times 10^{5}$ & 6.5 & $1.3 \times 10^{8}$ & 5
\end{tabular}

${ }^{a}$ Human recombinant isozymes, stopped flow $\mathrm{CO}_{2}$ hydratase assay method ( $\mathrm{pH}$ 7.5) (Nishimori et al., 2007)

${ }^{\mathrm{b}}$ Zebrafish recombinant enzyme, stopped flow $\mathrm{CO}_{2}$ hydratase assay method ( $\mathrm{pH}$ 7.5), this work.

AAZ, acetazolamide, 5-acetamido-1,3,4-thiadiazole-2-sulfonamide 


\section{Table 3 (on next page)}

Relative expression ratios of ca6 mRNA in adult zebrafish tissues. 
1 Table 3. Relative expression ratio of ca6 mRNA in adult zebrafish tissues

2

\begin{tabular}{lr}
\hline Tissue & $\begin{array}{c}\text { Relative } \\
\text { expression }\end{array}$ \\
\hline Fin/tail & 214.82 \\
Teeth & 6.81 \\
Spleen & 2.37 \\
Kidney & 19.44 \\
Brain & 293.46 \\
Swim bladder & 0.61 \\
Heart & 0.01 \\
Gills & 68.70 \\
Skin & 4.32 \\
Intestine & 0.41 \\
Pancreas & 0.27 \\
Eggs & 0.01 \\
Liver & 0.02 \\
\hline
\end{tabular}

3 


\section{Table 4(on next page)}

Statistics of swimming pattern analysis of ca6 morphant and wild-type zebrafish. 
1

2 Table 4. Statistics of swimming pattern analysis of ca6 morphant and wild-type zebrafish

\begin{tabular}{|c|c|c|c|c|c|}
\hline $\mathrm{A}^{\mathrm{a}}$ & Median & Mean & SD & Range & p-value \\
\hline \multicolumn{6}{|c|}{ Day 4} \\
\hline $\mathrm{KD}$ & 0.00 & 1.87 & 6.59 & $0.00-49.03$ & $4.28 \times 10^{-19 b}$ \\
\hline WT & 13.80 & 13.12 & 6.01 & $0.00-25.45$ & $1.90 \times 10^{-3} \mathrm{c}$ \\
\hline \multicolumn{6}{|c|}{ Day 5} \\
\hline $\mathrm{KD}$ & 4.75 & 4.89 & 4.26 & $0.00-5.19$ & $1.16 \times 10^{-7} \mathrm{~b}$ \\
\hline WT & 10.22 & 10.38 & 3.18 & $1.83-17.27$ & \\
\hline$B^{d}$ & Median & Mean & SD & Range & p-value \\
\hline \multicolumn{6}{|c|}{ Day 4} \\
\hline $\mathrm{KD}$ & 0 & 9.13 & 20.46 & $0.00-60.00$ & $8.68 \times 10^{-11 b}$ \\
\hline WT & 31 & 29.59 & 22.44 & $0.00-60.00$ & $4.98 \times 10^{-3} \mathrm{c}$ \\
\hline \multicolumn{6}{|c|}{ Day 5} \\
\hline $\mathrm{KD}$ & 24.51 & 26.96 & 26.23 & $0.00-60.00$ & $2.98 \times 10^{-4} \mathrm{~b}$ \\
\hline WT & 51.47 & 45.29 & 16.42 & $0.00-60.00$ & \\
\hline
\end{tabular}

KD: knockdown; WT: wild type

a Swimming distances $(\mathrm{cm})$

${ }^{\mathrm{b}} \mathrm{KD}$ compared to WT

c Day 4 WT compared to Day 5 WT

$\mathrm{d}$ Time spent in upper half of the flask 


\section{Figure 1 (on next page)}

Bayesian phylogenetic tree of CA VI, CA IX, CA XII, and CA XIV.

Analysis of protein alignment guided DNA alignments as detailed in Materials and methods.

Sidebars indicate the groups of isoforms. The CA VI subtree with more species is shown in Figure S2. 


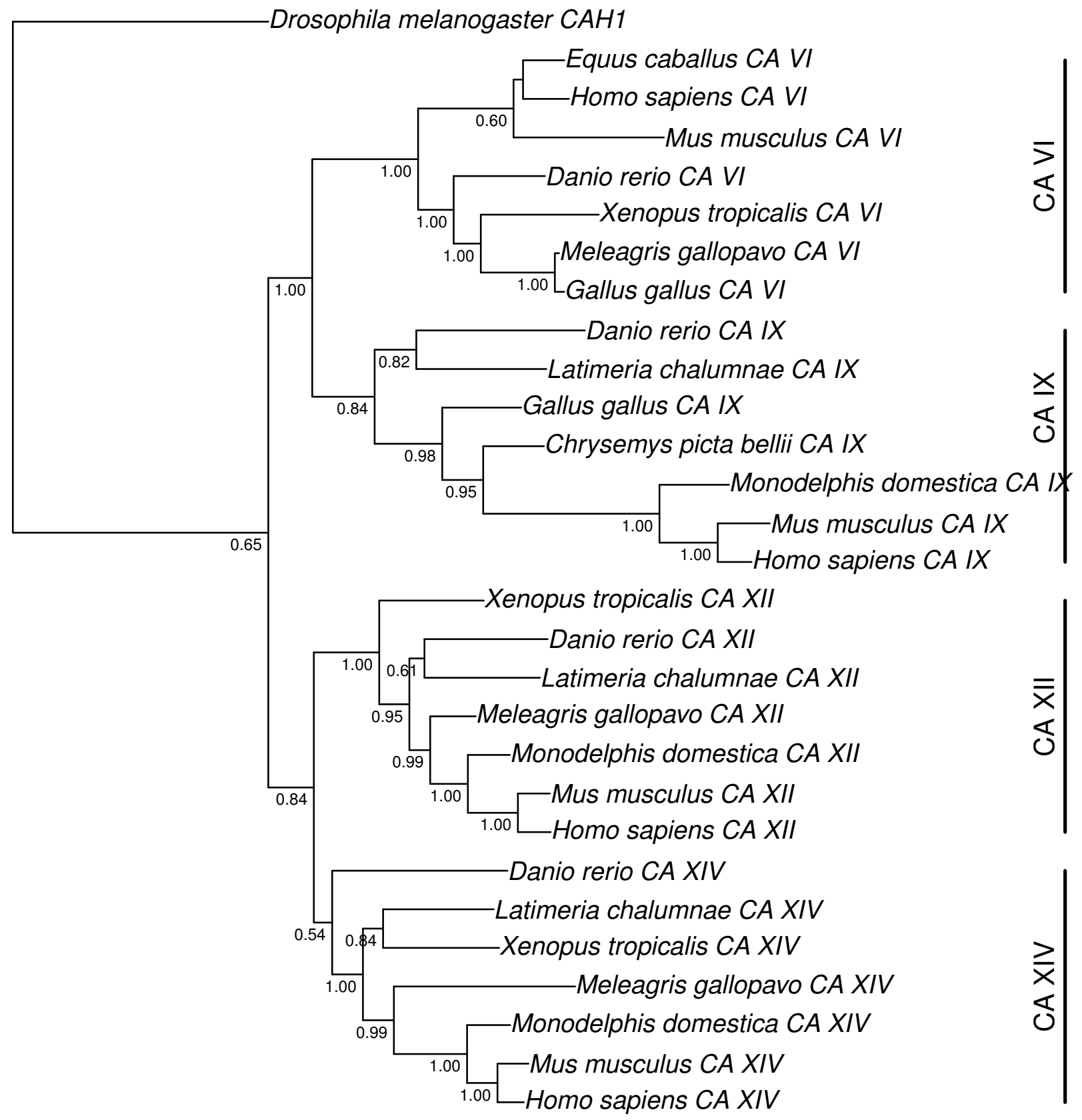




\section{Figure 2 (on next page)}

Bayesian phylogenetic tree of pentraxin domains.

Analysis of protein alignment guided DNA alignments as detailed in Materials and methods. Sidebars indicate PTX domains extracted from non-mammalian CA VI sequences (bottom) and groups of human pentraxins. 


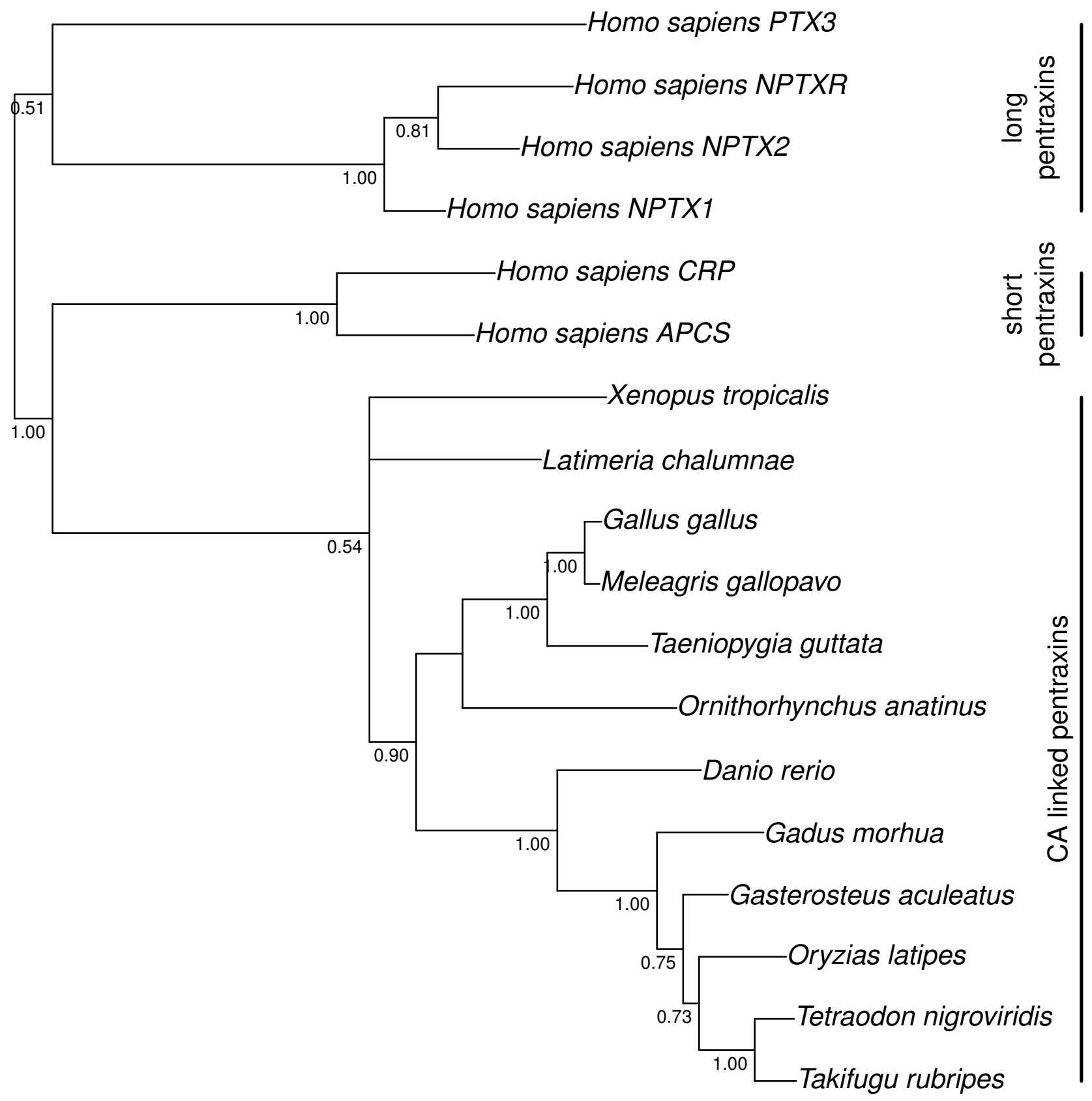




\section{Figure 3}

Zebrafish wild type and ca6 knockdown movement analysis.

Boxplots from video analysis of one minute of swimming of zebrafish larvae. KD: knockdown; WT: wild-type. Same data used for both A and B. Statistics of both analyses are given in Table 3. A) Total distances traveled. B) Time spent in the upper half of the tank (seconds, out of $60 \mathrm{~s})$. 

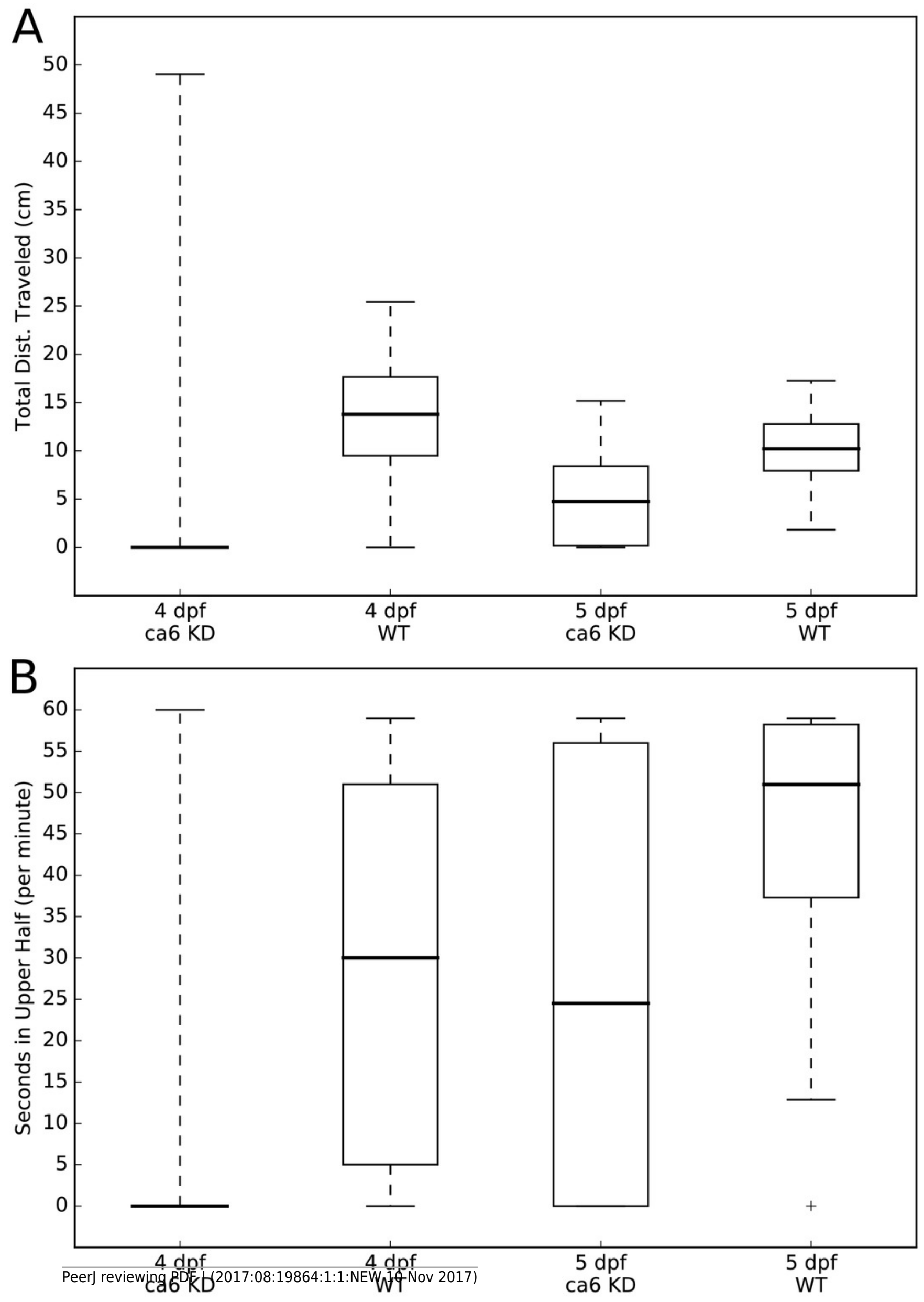


\section{Figure 4}

Amphipathic helix analysis in CA VI.

A) Helical wheel diagram of human CA VI (287 to 303). B) Helical wheel diagram of zebrafish CA VI (293 to 310). Multiple sequence alignment of the spacer region of CA VI from indicated species C). CA indicates the end of the catalytic CA domain; APH is the suggested amphipathic helix, which is analyzed in panels $A$ and B; and PTX indicates the approximate start of the pentraxin domain (not applicable to $H$. sapiens CA VI).

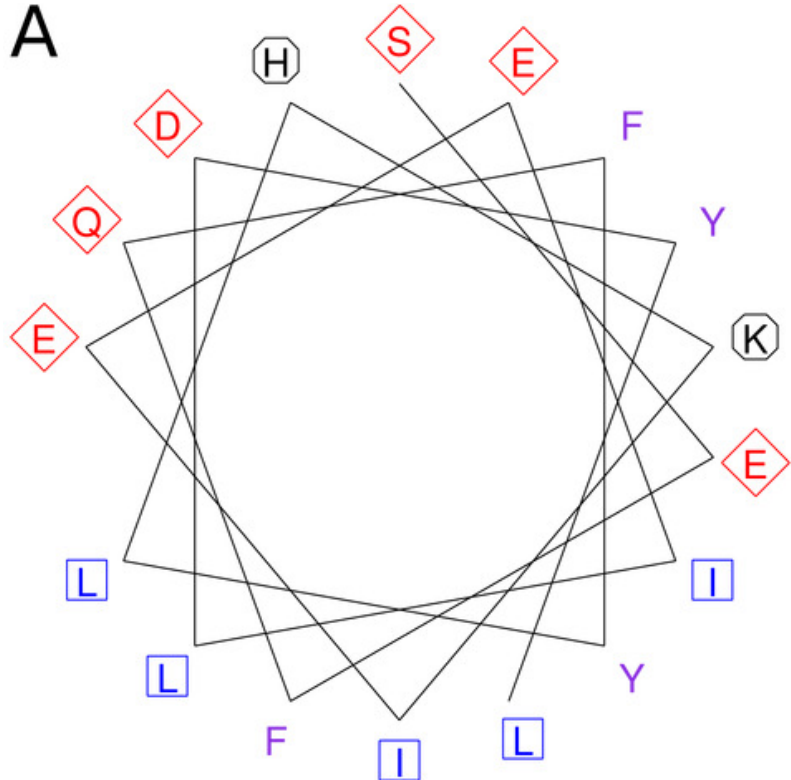

SEFQFYLHKIEEILDYL

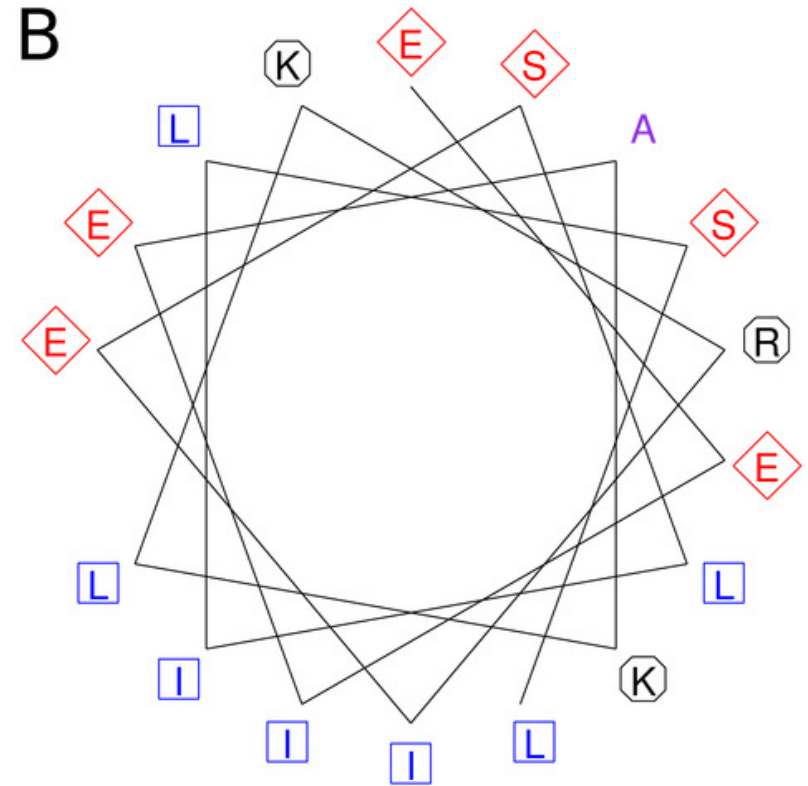

EEIEAKLKRIESLILSL

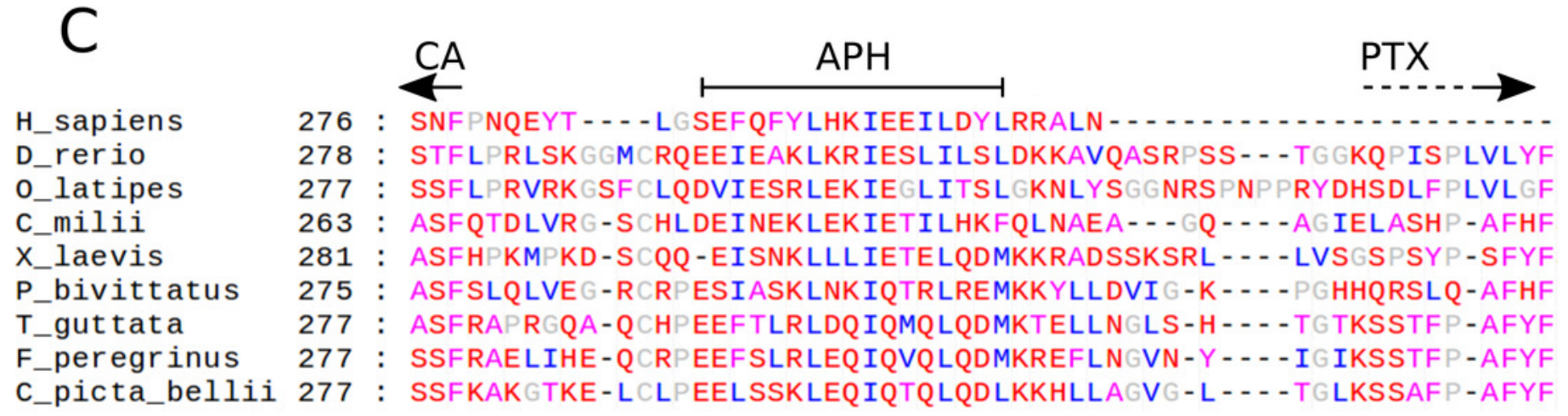




\section{Figure 5}

\section{Molecular models of zebrafish CA VI-PTX.}

A) One protomer shown in two orientations, CA domain at the top, PTX at the bottom.

Potential glycosylation site Asn residues are shown as spheres, active-site histidines and assumed disulfide cysteines as sticks. B) Front view of the pentamer model. In B to D, individual protomers are shown in different colors. Asn residues in glycosylation sites and active-site histidines are shown in spheres, cysteines not highlighted. C) Back view of the pentamer model. D) Side view of the pentamer model, seen from the top of panel C, with back view downwards.

*Note: Auto Gamma Correction was used for the image. This only affects the reviewing manuscript. See original source image if needed for review. 


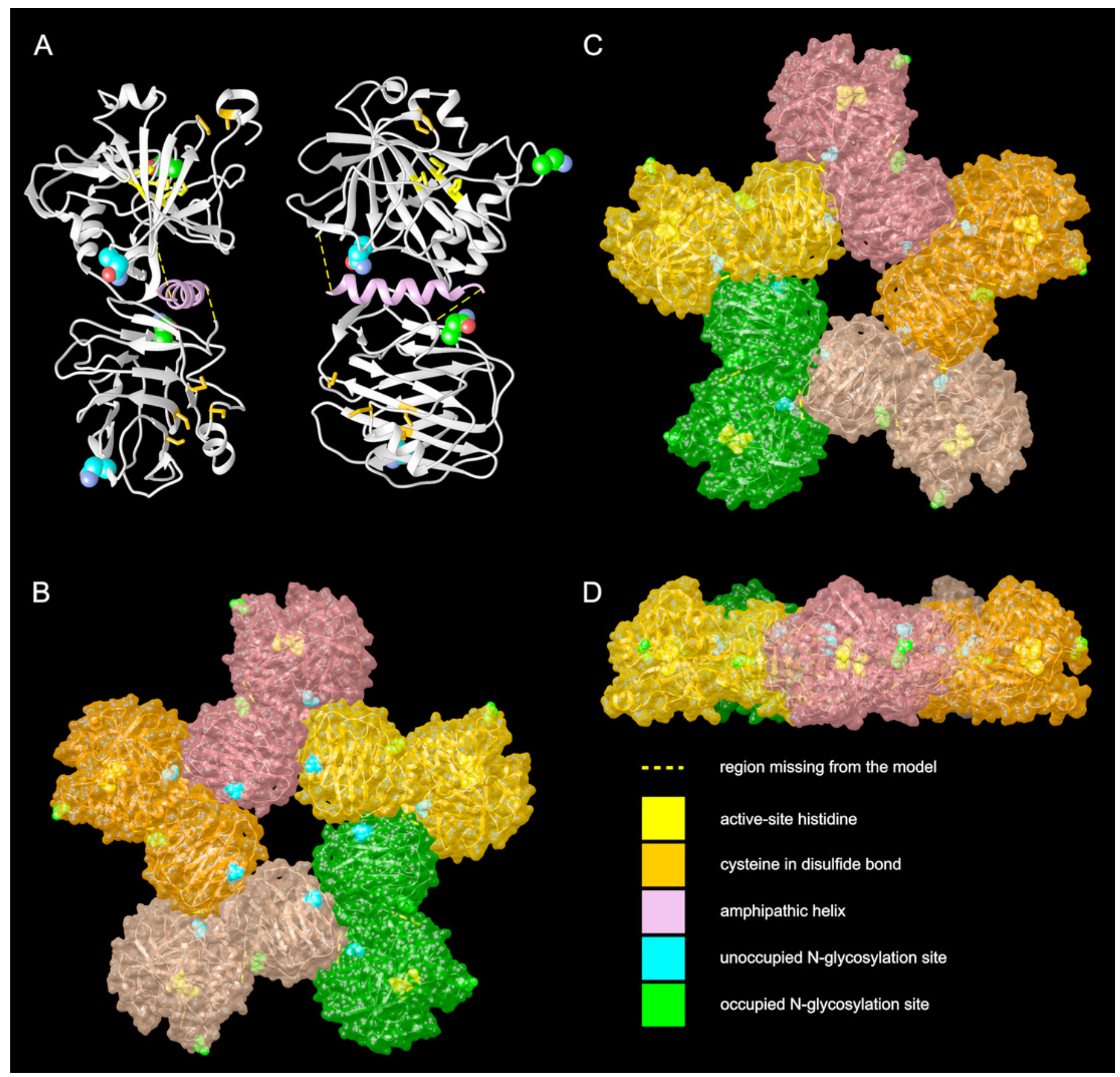


Figure 6

SDS-PAGE of recombinantly produced zebrafish CA VI-PTX.

Left: purified recombinant zebrafish CA VI-PTX, molecular mass calculated from mobility 58.6

kDa. Right: molecular weight standards. 
$\mathrm{kDa}$

250

150

100

75

50

37 


\section{Figure 7 (on next page)}

Assessment of the oligomeric size of zebrafish CA VI.

Gel permeation chromatography was used to study the characteristics of recombinantly produced zebrafish CA VI. The left Y-axis shows the UV absorption intensity (280 nm wavelength) and light scattering (LS) intensity. UV intensity was used for the determination of the protein concentration. Molecular weight (MW) was calculated using LS intensity and shown on the right $Y$-axis. Hydrodynamic radius $\left(R_{h}\right)$ was calculated from the dynamic light scattering signal, and is also shown on the right Y-axis. In addition, the oligomeric size of zebrafish CA VI was evaluated based on the penetration time using molecular weight marker proteins as a standard. 


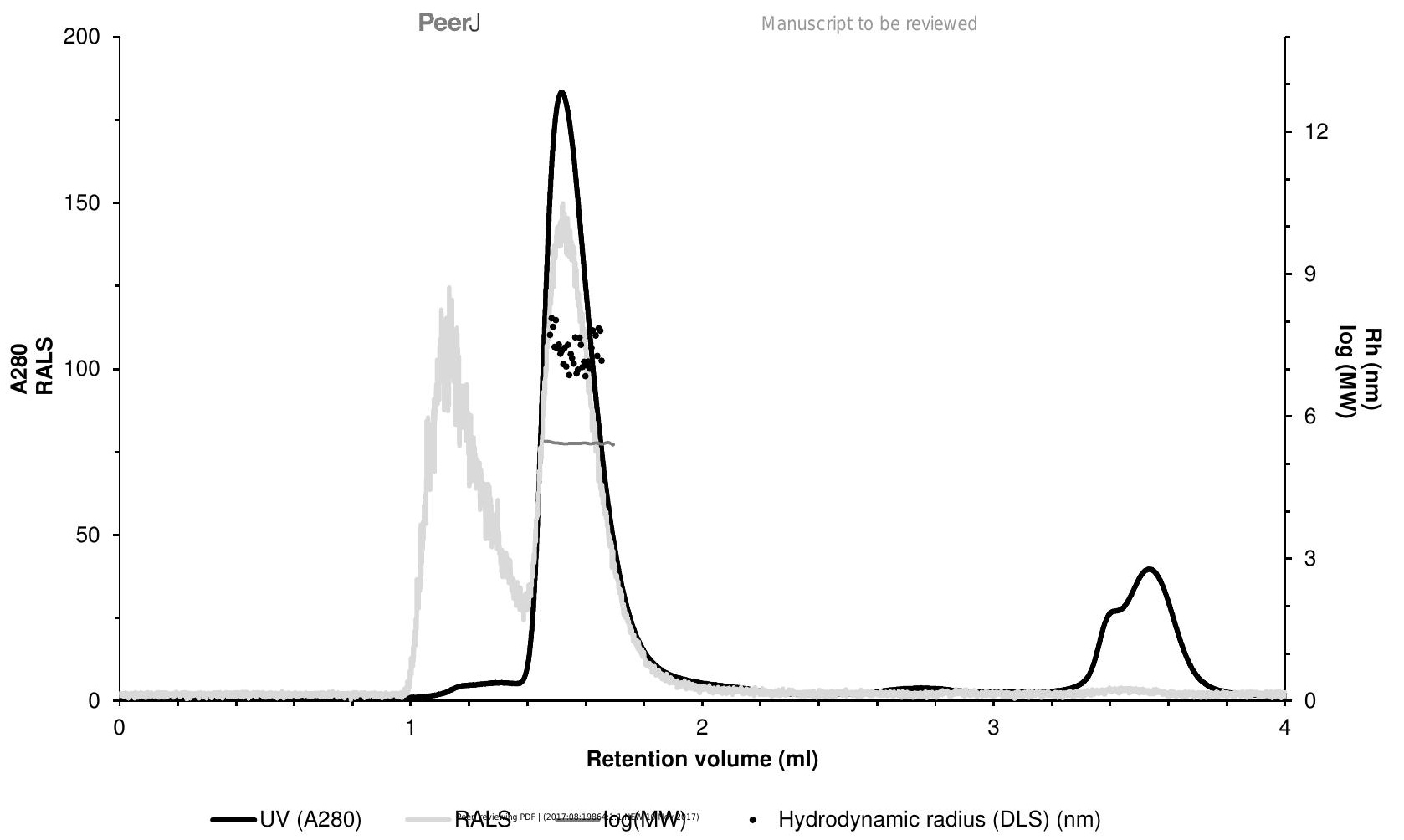




\section{Figure 8 (on next page)}

High-resolution mass spectrum of the tryptic digest of CAVI-PTX.

The mass spectrum was measured by direct infusion on a 12-T Fourier transform ion cyclotron resonance instrument using positive-ion electrospray ionization. Monoisotopic $\mathrm{m} / \mathrm{z}$ values and charge states obtained through peak deconvolution are indicated for the most abundant peaks. 
$2+$

707.9346

$2+$

765.4405

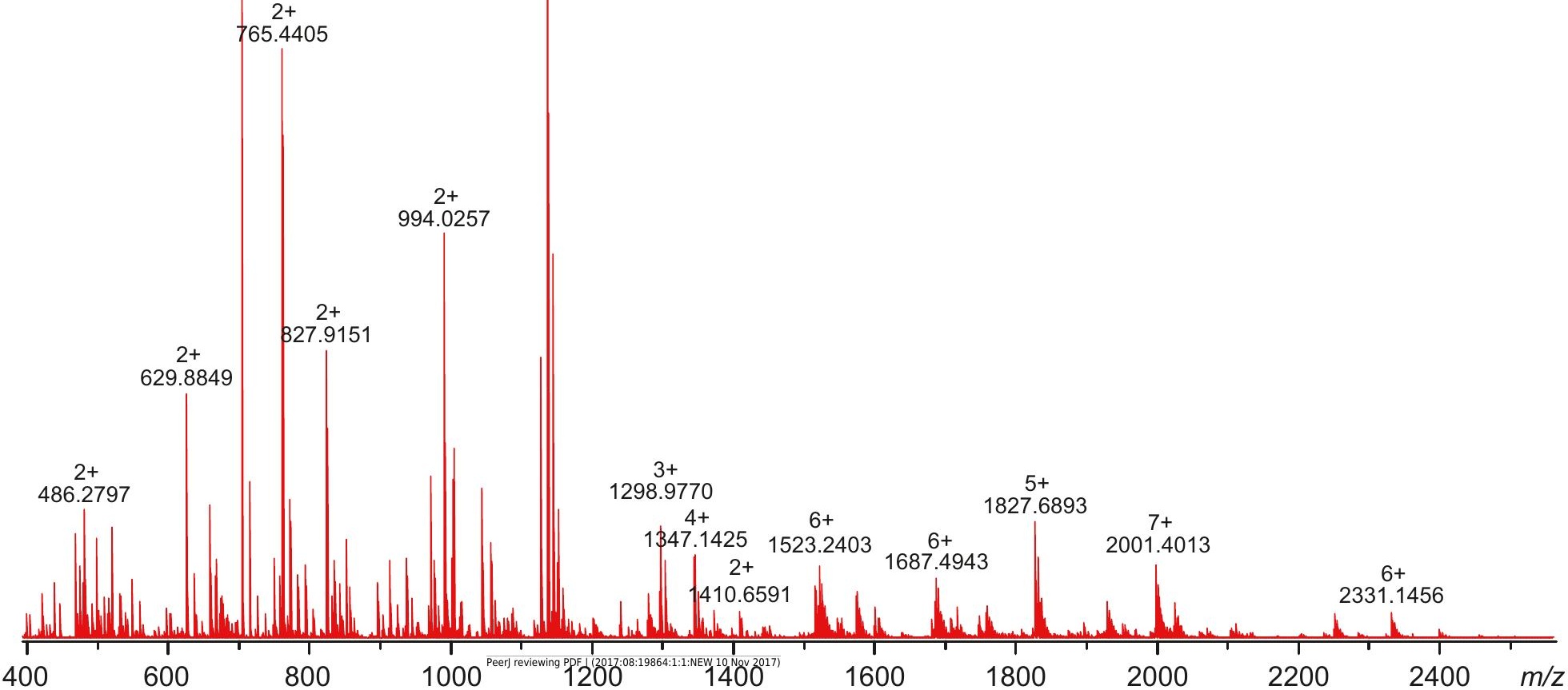




\section{Figure 9 (on next page)}

A tryptic peptide map of selected peptides of zebrafish CA VI-PTX.

The identified tryptic peptides are indicated with blue boxes showing the start and the end residues. The confirmed disulfide bonds are indicated with red lines with the corresponding peptides indicated with red boxes. The four potential $\mathrm{N}$-glycosylation sites are indicated with a distinct background color (blue: unoccupied N-glycosylation site; and green: occupied Nglycosylation site). The three observed glycopeptides are marked with purple boxes. The start of the PTX domain (KQP...) has been indicated with a black arrow. This figure shows a minimum amount of peptides for maximal coverage, whereas all identified peptides are shown in Fig. S6. 


\begin{tabular}{|l|r|}
\hline 20-35erJ & $36-5 \$+217-247$ \\
\hline \hline $20-56+219-247$ & \\
\hline & \\
\cline { 2 - 2 } &
\end{tabular}

90 EIQLPSTMKITKGFPHQYTAVQMHLHWGGWDLEASGSEHTMDGIRYMAELHVVHYNSEKYPSFEEAKNKP

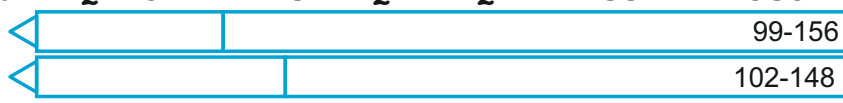

$02-148$

$135-156$

160 DGLAVLAFFFEDGHFENTYYSDFISNLANIKYVGQSMSISNLNVLSMLSENLSHFYRYKGSLTTPPCFES 157-190 191-216 $36-56+217-247$ $20-56+219-247$

230 VMWTVFDTPITLSHNQIRKLESTLMDHDNKTLWNDYRMAQPLNERVVESTFLPRLSKGGMCRQEEIEAKL

\begin{tabular}{|l|l|l|l|}
\hline & $248-266$ & $267-274$ & $275-286$ \\
\hline & \multicolumn{3}{|c|}{$248-283$} \\
\hline
\end{tabular}

300 KRIESLILSLDKKAVQGKQPISPLVLYFPQKNVESFAVVNLTHPMELKSFTACMNVQIPPIRDLTVLSYS

\begin{tabular}{|c|c|c|c|c|}
\hline & $313-330$ & $331-347$ & & \\
\hline & \multicolumn{3}{|c|}{$313-430$} & \\
\hline $301-312$ & $312-330$ & & $331-42 \$$ & \\
\hline
\end{tabular}

370 TSHDNELMISLGSEVGLWIGDEFVNLSFDLPSSDWTNYCLTWASHNGGAELWVNGVVGKERYIRTGYIIP

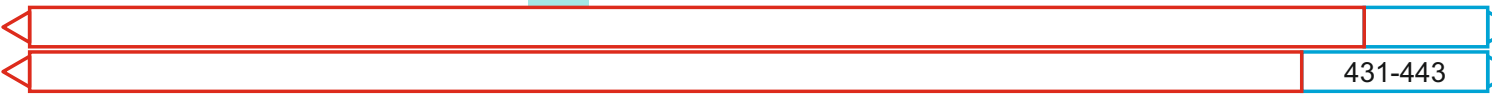

440 AGGRLILGKDQDGFLGISVNDAFVGHMSDVNIWDYVLTEGEIVEQMSCDNGKVKGNVLSWGVTQLSLYGG

\begin{tabular}{|l|l|l|}
\hline $434-443$ & $444-491+494-520$ & \\
\hline & $444-491+494-526$ & \\
\hline & & \\
\cline { 1 - 2 } & &
\end{tabular}

\begin{tabular}{|l|}
\hline $444-491+494-520$ \\
\hline \hline $444-491+494-526$ \\
\hline
\end{tabular}

510 VQLQGEQVCHRDNNNNRETEKLVPR

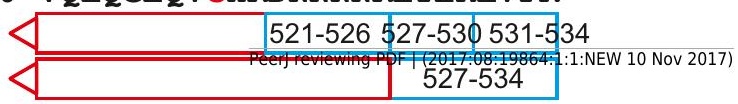


Figure 10 (on next page)

Characterization of zebrafish CA VI-PTX glycopeptides by tandem mass spectrometry.

The precursor ions of the two observed glycopeptides, with monoisotopic masses of 3416.5084 Da and 2819.3059 Da (residues 248-266 and 331-347, respectively), were massselected in a quadrupole for collision-induced dissociation tandem mass spectrometry. The fragmentation patterns are consistent with the presence of the standard N-glycosylation core pentasaccharide with fucosylation in the innermost $\mathrm{N}$-acetylglucosamine residue in the glycopeptide 248-266 (A) and a similar non-fucosylated pentasaccharide in the glycopeptide 331-347 (B). 
CID spectrum from KLESTLMDHDNKTLWNDYR + 2x GIcNAc + 3x Man + 1x Fuc (m/z 855.1335, 4+)

PeerJ $818.6191(4+)$

Fuc

$-146.0576$

$814.6204(4+)$

Man

- 162.0524

$778.1060(4+)$

Man

$-162.0524$

$774.1071(4+)$

Man

$-162.0532$

$855.1335(4+)$

Manuscript to be reviewed

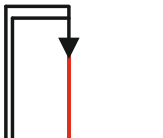

\begin{tabular}{c||c||c|c|}
$737.5928(4+)$ \\
Man
\end{tabular}

$-162.0492$

$733.5937(4+)$

Man

$-162.0536$

$528.1922(1+) \underset{\text { Man }}{690.2450}(1+)$
-162.0528 $-162.0528$

600

$861.4104(3+)$

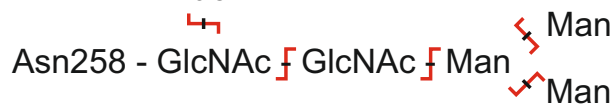

CID spectrum from NVESFAVW LTHPMELK + 2x GIcNAc + 3x Man $\begin{array}{r}1167.5792(2+) \\ (\mathrm{m} / \mathrm{z} 946.7746,3+)\end{array}$

$1066.0397(2+)$

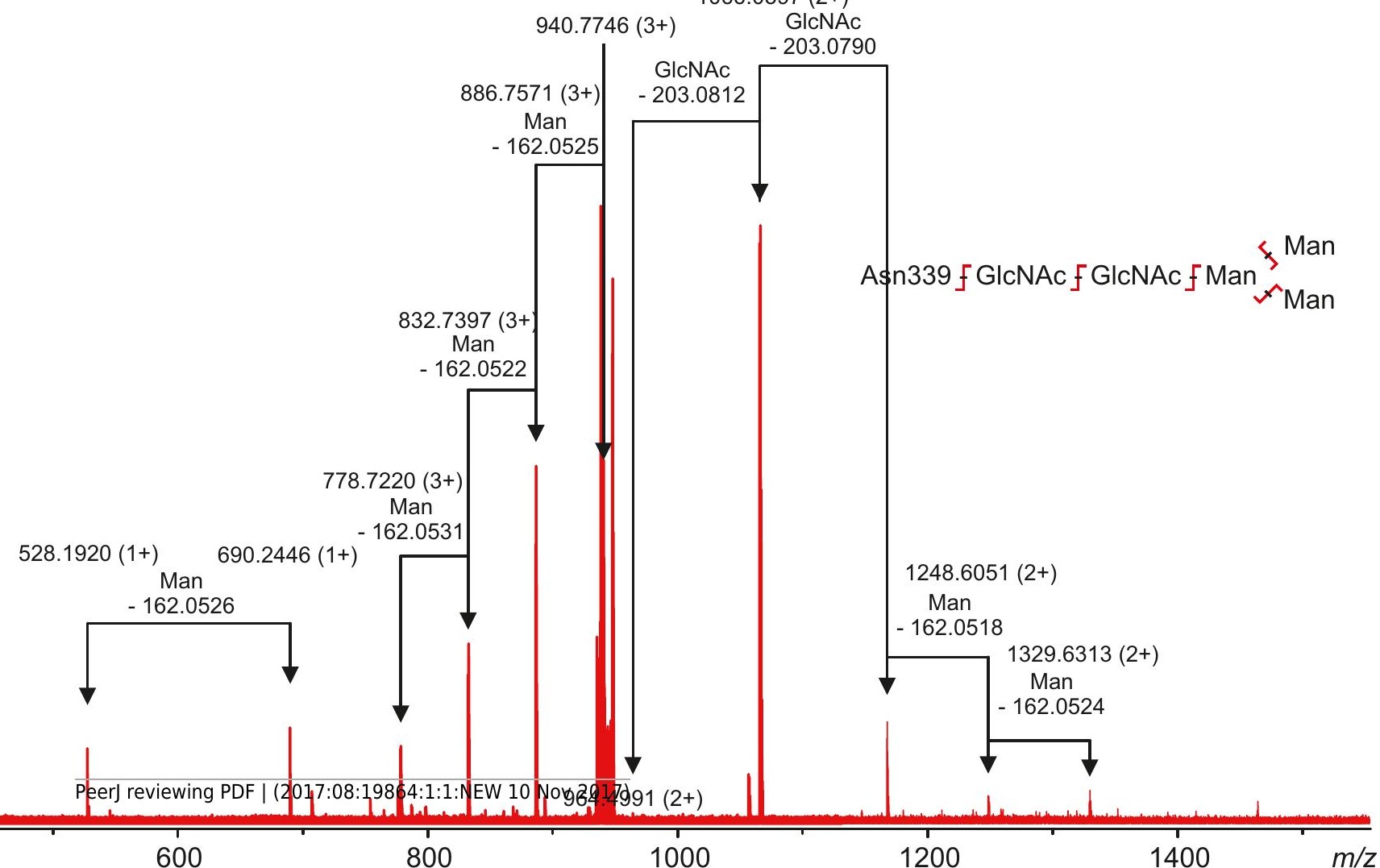




\section{Figure 11}

Immunohistochemistry of CA VI-PTX in adult zebrafish tissues.

Tissue sections stained by anti-zebrafish CA VI-PTX (green) and nuclear staining by DAPI (blue). Gills (A), heart (B), skin (C), swim bladder (D). The strongest signal (A-D) is present on the cell surfaces, even though the cell interior gives some background staning. Scale bars $100 \mu \mathrm{m}$.

*Note: Auto Gamma Correction was used for the image. This only affects the reviewing manuscript. See original source image if needed for review. 

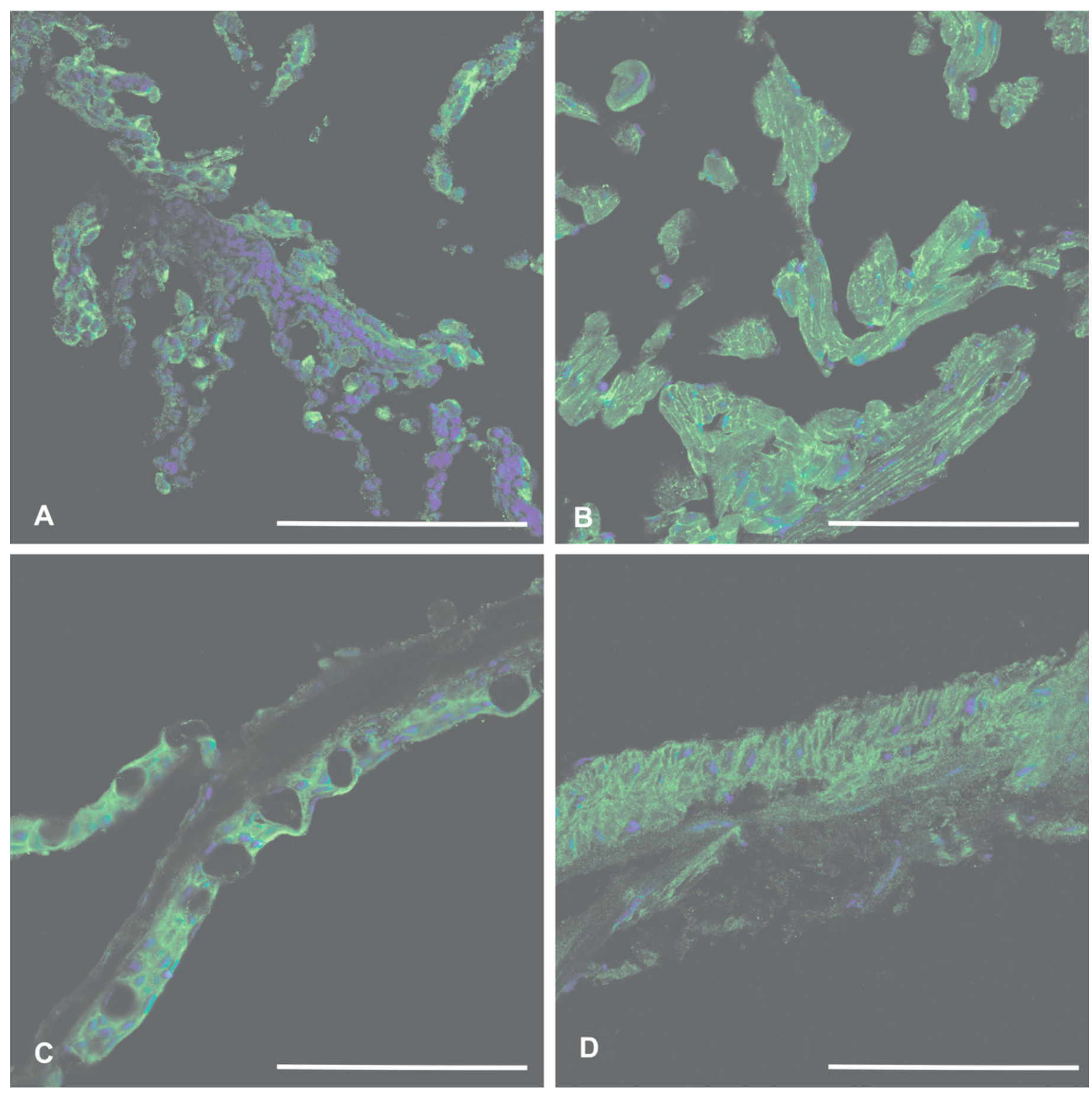


\section{Figure 12}

Comparison between morpholino injected and wild-type zebrafish larvae.

The morphant larvae (A) showed consistently a deflated swim bladder at $4 \mathrm{dpf}$ (arrow), which returned to normal morphology at $5 \mathrm{dpf}$. Wild-type larvae of the same ages are shown for comparison (B). Scale bars $1 \mathrm{~mm}$.

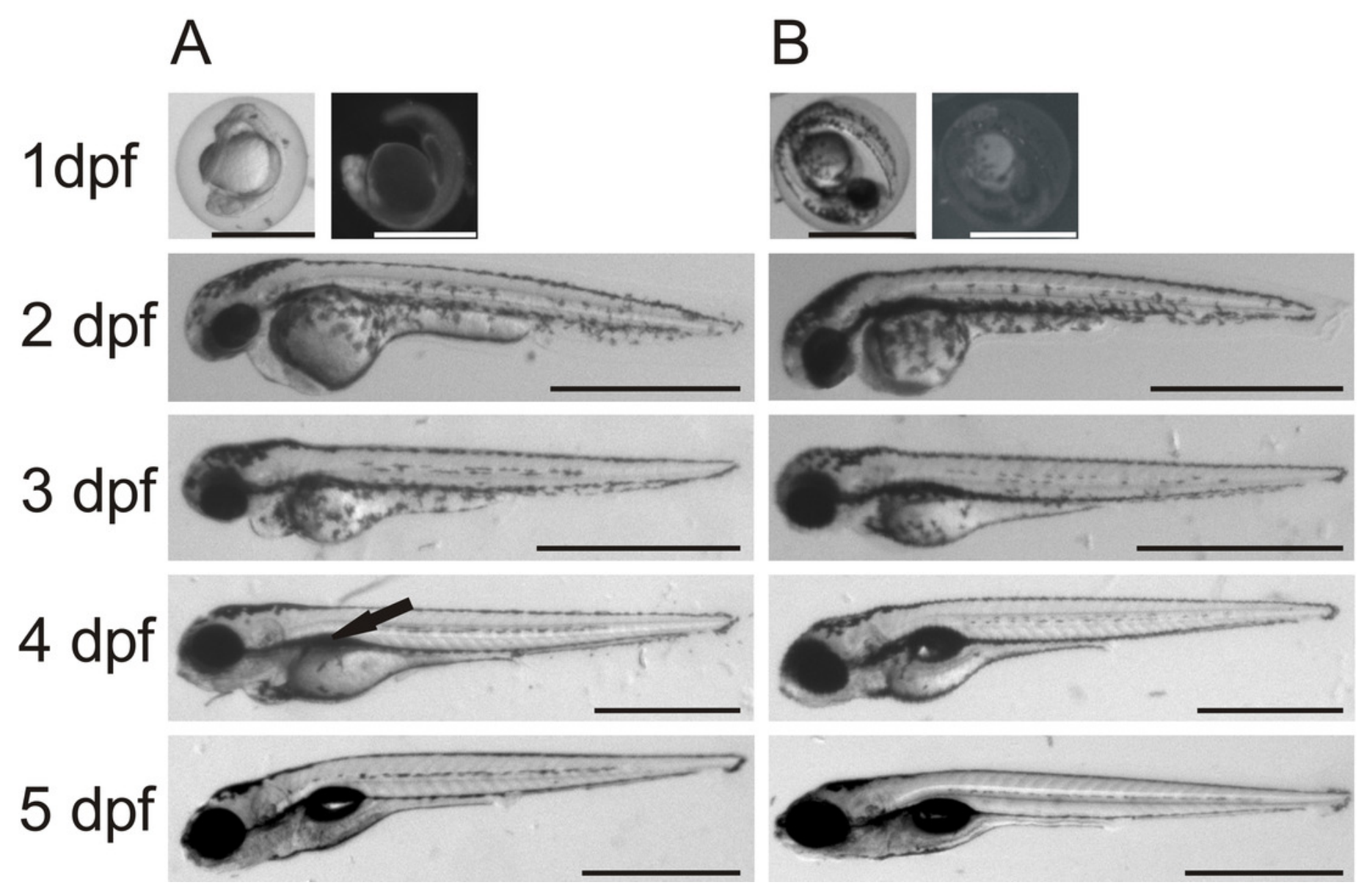


Figure 13 (on next page)

Developmental expression pattern of ca6 in 1 to $5 \mathrm{dpf}$ larvae.

The expression levels of the ca6 gene was studied using qRT-PCR from the total mRNA isolated from 1-5 dpf of ca6 morphant and wild-type larvae. The results of ca6 gene expression were normalized using gapdh as internal control. 


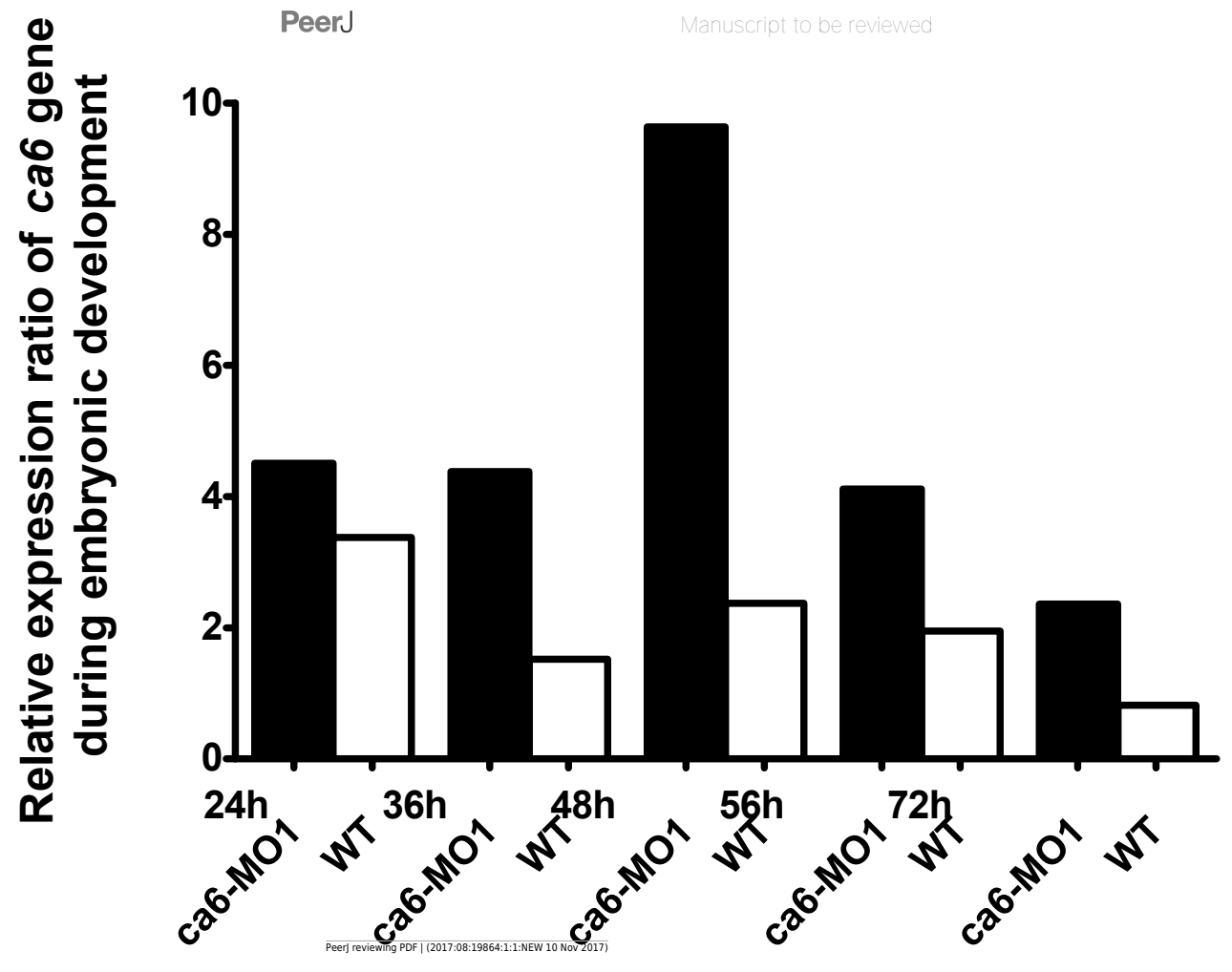


Figure 14(on next page)

Hypothesis of evolution of the domain composition in CA VI and the transmembrane CA isoforms.

$\mathrm{CA}=$ catalytic $\mathrm{CA}$ domain; $\mathrm{TMH}=$ transmembrane helix; $\mathrm{APH}=$ amphipathic helix; $\mathrm{PTX}=$ pentraxin domain; $P G=$ proteoglycan domain. Image credit: Original digital art by Jukka Lehtiniemi. 


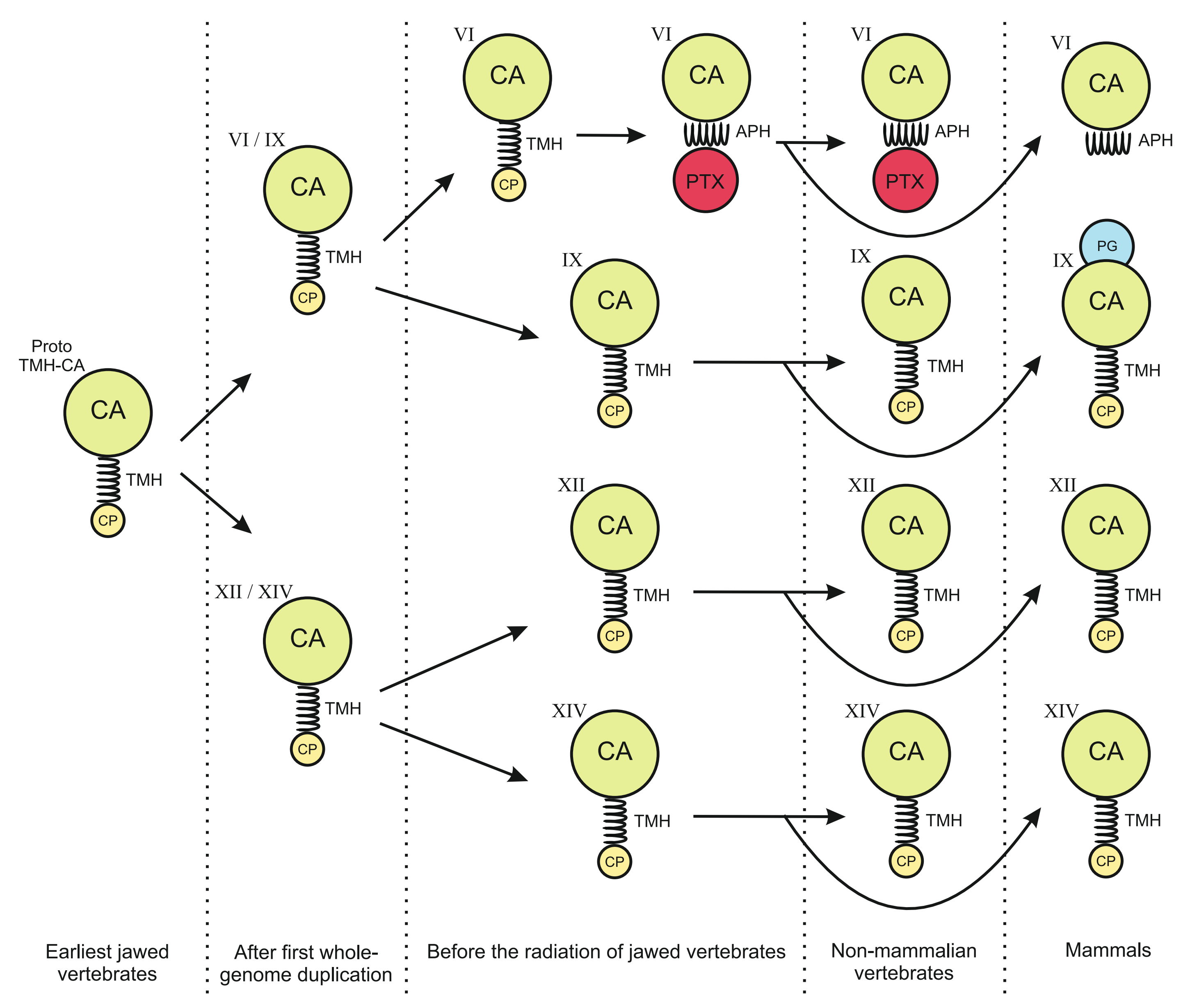

